\title{
Ueber den blutdruckerregenden Bestandtheil der Nebenniere, das Epinephrin.
}

\author{
Von
}

John J. Abel.

(Aus dem pharmakologischen Institut der Johns Hopkins University, Baltimore.)

(Der Redaction zugegangen am 2'x. Juli 1899.)

Es ist etwa ein halbes Jahrhundert her, dass die grosse Wichtigkeit der Nebenniere erkannt wurde, und seither hat dieses Organ in hervorragendem Grade die Aufmerksamkeit der medicinischen Welt auf sich gezogen.

Im Jahre 1855 beschrieb Addison ${ }^{1}$ ) die allgemeinen und lokalen Wrirkungen der Erkrankung der Nebenniere und fügte die Morbus Addisonii zur Reihe der in ihren Ursachen erkannten Krankheiten. Ein Jahr später beobachtete Vulpian, ${ }^{2}$ ) dass der ausgepresste Saft dieses Organs von verschiedenen Thieren sich in charakteristischer Weise gegen Eisenchlorid und Jodlösung verhielt und nirgends. anders im Thier ähnliche Reactionen zu erhalten sind. Zur selben Zeit zeigte BrownSéquard, ${ }^{3}$ ) dass die Entfernung der Nebennieren aus dem Thier den Tod nach sich zieht.

Im Jahre 1894 fanden Schäfer und Oliver ${ }^{4}$ ) und bald darauf Scymonowicz und Cybulski, ${ }^{5}$ ) dass schon eine sehr geringe Dosis des wässerigen Nebennierenextracts hinreicht,

1) Dr. Addison's Works, New Sydenham Society (1868), S. 211.

2) Compt. rendus. Acad. d. sc., Paris, Bd. 93 (1856), S. 663-665.

3) Ibid. (18566), S. 422, 542.

4) Journal of Physiology, Bd. 16 (1894), Bd. 18 (1895).

5) N. Cybulski: Weitere Untersuchungen über die Function der Nebenniere. Anz. d. Akad. d. Wissensch. in Krakau, 4. März 1895, citirt aus L. Sczymonowiczz Arch. f. die gesammte Phisiol., Bd. 64, S. 149. 
den Blutdruck sehr bedeutend $\mathrm{zu}$ erhöhen, was seither von mehreren andern Beobachtern bestätigt wurde. Schäfer und Oliver ${ }^{-1}$ ) haben ferner festgestellt, dass bei weitgehender Degenerirung des Markes der Nebenniere das Extract sämmtliche physiologische Wirkungen eingebüsst hat.

Auch die Isolirung des wirksamen Bestandtheils und die Feststellung seiner chemischen Eigenschaften bildeten den Gegenstand von Untersuchungen. Vulpian und Cloëz, ${ }^{2}$ ) Virchow, ${ }^{3}$ ) Arnold, ${ }^{4}$ ) Seligsohn, ${ }^{5}$ ) Holm, ${ }^{6}$ ) Krukenberg, ${ }^{7}$ ) Brunner, ${ }^{8}$ ) Radziejewsky, ${ }^{9}$ ) Mühlmann, ${ }^{10}$ ) Metzger, ${ }^{11}$ ) Gürber, ${ }^{12}$ ) Fraenkel, ${ }^{13}$ ) Moore ${ }^{14}$ ) und v. Fürth ${ }^{15}$ ) mögen in dieser Beziehung erwähnt werden. Ich habe bereits in früheren Publicationen mehrere dieser früheren und neueren Arbeiten beleuchtet und einige der neueren Abhandlungen wurden auch von 0 . v. F ürth ${ }^{16}$ ) kritisch besprochen. Aber so vortrefflich auch viele der Abhandlungen waren, haben sie doch wichtige Fragen nicht aufgeklärt und manche frïhere Schlussfolgerungen wurden seither als irrig erkannt. Das Problem bot grosse Schwierigkeiten dar.

In meiner ersten Abhandluung, der Association of American Physicians, mitgetheilt am 6. Mai 1897, und in Johns Hopkins Hospital Bulletin vom Juli 1897 veröffentlicht, habe ich be-

1) Loc. cit., Bd. 18 (1895), S. 269.

2) Compt. rend., Acad. d. sc., Paris (1857), Bd. 94, S. 340-343.

3) Virchow's Archiv, Bd. 12 (1857), S. 481-483.

4) Ibid., Bd. XXXV (1866), S. 64-107.

5) Ibid., Bd. XVIII (1860), S. 35்̃.

6) Journ. f. pract. Chemic, Bd. 100 (1867), S. 150.

7) Virchow's Archir, Bd. 101 (1885), S. 5́42-591.

8) Schweizer. Wochenschr. f. Pharm., Bd. 30, 1892, S. 121-12:3.

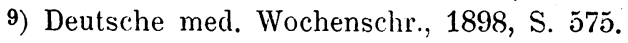

10) Deutsche med. Wochenschr., 1896, Nr. 26, S. 409-411.

11) Zur Kenntniss der wirksamen Substanzen der Nebennieren.

Diss. Würzburg 1897.

12) Münch. med. Wochenschr., 1897, S. 750.

13) Wiener med. Blätter, 1896, Nr. 14-16.

14) Journal of Physiology, vol. 21, No. 4 and 5 (1897).

15) Diese Zeitschr., Bd. XXIV, S. 142.

16) Ibid., Bd. XXVI, S. 15. 
wiesen, dass die blutdrucksteigernde Substanz weder Brenzkatechin ist, wie Mühlmann vermuthet hat, noch ein unmittelbares Derivat davon, obwohl ich zugab, dass unter den Produkten der Fäulniss des Saftes sich etwas Brenzkatechin befinden könnte. Mühlmann hat die Prüfung auf Brenzkatechin nicht vollständig durchgeführt. Obgleich einige Reactionen darauf zu deuten scheinen, so fehlt doch vor Allem die Fähigkeit, reducirend auf Fehling's Lösung zu wirken. Auch unterscheidet sich die blutdrucksteigernde Substanz von Brenzkatechin durch seine Fähigkeit, Salze zu bilden, Farbenreactionen mit Alkaloidreagentien zu geben, sowie in anderer Weise. Sowohl v. Fürth als auch Metzger haben, ohne Kenntniss von meinen Arbeiten zu haben, meine Ansicht ebenfalls bestätigt. Wir können sicher sein, Brenzkatechin ist nicht in der Nebenniere vorhanden.

Ich habe bereits in meiner erwähnten Abhandlung gezeigt, dass man bei Benzoylirung nach Schotten-Baumann den wirksamen Stoff der Nebennieren aus seiner wässerigen Lösung abscheiden kann. Das Filtrat von dieser Benzoylverbindung gibt keine charakteristische Reactionen mehr, und anstatt Erhöhung des Blutdrucks wird im Gegentheil ein Sinken beobachtet. Aus der erwähnten Benzoylverbindung konnte ich den wirksamen Bestandtheil mit allen seinen charakteristischen Eigenschaften regeneriren und schliesslich ein noch unreines Sulfat des sich wie ein Alkaloid verhaltenden Körpers darstellen. In einer zweiten Abhandlung, veröffentlicht in der September-October-Nummer des Johns Hopkins Hospital Bulletin 1898, habe ich ausser weiterer Bestätigung und Vervollständigung meiner früheren Befunde mitgetheilt, dass es mir gelungen ist, das blutdrucksteigernde Princip in Form reiner Salze zu isoliren.

\section{Epinephrin.}

Hyrtl1) hat für den Ausdruck "Nebenniere» die Bezeichnung Epinephris vorgeschlagen, da dieses Organ mit der Niere gar nichts zu thun hat. Ich nenne daher die blut-

1) Lehrbuch der Anatomie des Menschen, 17. Aufl., S. 775. 
drucksteigernde Substanz in Uebereinstimmung mit Hyrtl's Nomenclatur Epinephrin. Ich habe die Ueberzeugung, dass ein gründlicheres Studium dieser Verbindung Licht auf die Symptome der Addison'schen Krankheit werfen wird, Symptome, welche in vielen Beziehungen an Zustände erinnern, die wir als Autointoxicationen bezeichnen.

\section{Benzoylderivat des Epinephrins.}

Ich habe bereits in meiner zweiten Abhandlung ausführlicher die Bereitung des rohen Benzoylproduktes beschrieben. Nachdem ich verschiedentlich versucht hatte, bessere Methoden zu finden, bin ich auf meine frühere Methode zurückgekommen, habe jedoch beobachtet, dass es gerathen ist, höher concentrirte Extracte bei der Benzoylirung zu vermeiden. Je verdünnter die Lösungen, desto freier ist das Produkt von fremden Benzoaten. Ich verwendete meist Extracte von der Stärke, dass $20 \mathrm{l}$ das Epinephrin von $50 \mathrm{~kg}$ der Epinephris des Rindes enthielten. Obgleich das Epinephrin in der frischen Drüse nur zu etwa $0,01 \%$ enthalten ist, ist es dennoch leicht, jede Spur als Benzoylverbindung auszufällen. Ich habe es als zweckmässig befunden, Anfangs eine grössere Menge Benzoylchlorid zuzusetzen, dann nur wenig einer 10\% igen Aetznatronlösung, hierauf energisch zu schütteln und nach einigen Minuten wieder eine kleine Menge des Alkalis zuzusetzen und wieder zu schütteln, und dieses so oft zu wiederholen, bis die Rosafärbung nicht mehr erhalten wird.

Das Filtrat zeigt zwar bei weiterer Benzoylirung auch noch Niederschlïge; dieselben gehören jedoch andern Körpern an, über die ich später weitere Mittheilungen machen werde.

Dass durch die Benzoylirung das active Princip aus der Lösung entfernt wurde, ging aus folgender Probe hervor: Das Filtrat wurde nach Ansäuern mit Salzsäure von der Benzoesäure durch Ausschütteln mit Aether befreit und nach Neutralisation in kleiner Menge in die Jugularvene eines Hundes injicirt, worauf ein rasches und beträchtliches Sinken ${ }^{1}$ ) des

1) Bulletin of the Johns Hopkins Hospital, July 1897, p. 153. 
Blutdruckes folgte, also gerade das Gegentheil von der Wirkung des Epinephrins. Metzger ${ }^{1}$ ) hat auch beobachtet, dass die Nebenniere sowohl eine blutdruckerniedrigende als eine blutdrucksteigernde Substanz enthält, hält es aber für unentschieden, ob jene ein künstliches Produkt, entstanden durch die verschiedenen Manipulationen mit dem Nebennierenextract, oder ein vorgebildeter Bestandtheil ist.

Das nach meiner früheren Vorschrift ${ }^{2}$ ) gereinigte Benzoat bildet eine hellgelbe harzigẹ Masse von viel grösserer Beständigkeit als die Acetylverbindung und kann im Wasserbade beliebig lange erwärmt werden, ohne Spuren von Zersetzung zu erleiden. Es ist frei von Benzoylchlorid und Benzaldehyd, löst sich leicht in Alkohol und Essigäther, weniger leicht in Aethyläther, und kann durch Zusatz des letzteren partiell ausgefällt werden, worauf man eine weitere Reinigung basiren könnte.

Obgleich unter den beschriebenen Umständen wahrscheinlich nur eine Monobenzoylverbindung erhalten wird, kann doch noch nicht geschlossen werden, dass nur ein einziges ersetzbares Wasserstoffatom vorhanden sei, und es kann wohl sein, dass man weitere Benzoylgruppen einführen kann, wenn reines Epinephrinsalz mit Benzoylchlorid und Natriumalkoholat nach Claisen's Methode, ${ }^{3}$ ) oder mit Benzoesäureanhydrid und Natriumbenzoat nach Goldschmiedt und Hemmelmayr ${ }^{4}$ ) behandelt wird. Weiter unten wird gezeigt werden, dass ein Triacetylderivat erhalten werden kann, wenn das Epinephrinsulfat acetylirt wird.

Die verschiedenen Produkte, welche bei der Reinigung des rohen Benzoats entfernt werden, wurden noch nicht eingehend von mir untersucht. Es befindet sich darunter auch ein in schönen Prismen krystallisirendes Benzoylprodukt. Ueber ein Produkt von coniinartigem Geruch, welches bei der Benzoylirung bei Gebrauch von zu concentrirtem Alkali entsteht, habe ich früher bereits Andeutungen gemacht.

1) Loc. cit., S. 25 .

2) Bulletin of the Johns Hopkins Hospital, Sept.-Oct. 1898.

3) Berichte der deutschen chem. Ges. Bd. 27, S. 3181.

4) Monatshefte für Chemie Bd. 15, S. 327. 
Obgleich die Methode, das Epinephrin auf dem Wege über die Benzoylverbindung zu erhalten, etwas umständlich ist, gibt sie doch den besten Weg ab, zu den reinen Salzen zu gelangen. Da die Benzoylverbindung nicht krystallinisch zu erhalten ist, habe ich nur eine einzige Analyse ausgeführt.

$0,2966 \mathrm{~g}$ Substanz, bei $80^{\circ} \mathrm{C}$. über Schwefelsäure in Vacuo getrocknet, gaben $0,78895 \mathrm{~g} \mathrm{CO}_{2}$ und 0,14785 g $\mathrm{H}_{2} \mathrm{O}$. 0,29656 g Substanz von derselben Darstellung gaben $8,7 \mathrm{ccm}$. $\mathrm{N}$ bei $18.25^{\circ} \mathrm{C}$. und bei einem Barometerstand von $760 \mathrm{~mm}$.

$\begin{array}{cc}\text { Gefunden } & \text { Berechnet für } \mathrm{C}_{17} \mathrm{H}_{14} \mathrm{NO}_{4} \cdot \mathrm{CO} \cdot \mathrm{C}_{6} \mathrm{H}_{5} \\ \mathrm{C}=72,54 \% \% & \mathrm{C}=71,82 \% \\ \mathrm{H}=5,54 \% & \mathrm{H}=4,74 \% \\ \mathrm{~N}=3,46 \% \% & \mathrm{~N}=3,49 \%\end{array}$

\section{Spaltung der Benzoylverbindung.}

In meiner ersten Abhandlung habe ich mehrere Methoden mitgetheilt, nach denen ich die Spaltung der Benzoylverbindung vornahm. Ich löste z. B. das Produkt in Eisessig, erhitzte bis nahe zum Siedepunkt und goss in diese Lösung dann eine heisse 25\% ige Schwefelsäure zu gleichem Volumen in kleinen Portionen ein. Die Mischung wurde dann noch am Rückflusskühler 10 Minuten im Sieden erhalten. Später habe ich die Spaltung mit Wasser im Autoclaven unter einem Druck von 8-12 Atmosphären vorgenommen. Die resultirende Flüssigkeit gibt mit sehr verdünntem Ammoniak einen Niederschlag von Epinephrin, das dann direkt zur Herstellung von Salzen dienen kann. Obwohl die Benzoylverbindung selbst ganz aschefrei ist, so sind doch die auf diese Weise erhaltenen Produkte mit Mineralstoffen, herrührend aus den Gefässen, in welchen die Spaltung des Benzoylproduktes vorgenommen wurde, verunreinigt. Immerhin lieferten die freie Base und das Benzoat bei der Analyse Resultate, aus denen sich eine Grundlage zur Aufstellung der empirischen Formel ergab.

Ich habe kürzlich gefunden, dass bei Zusatz von $1-2 \%$ Schwefelsäure die Spaltung im Autoclaven schon bei 3 bis 5 Atmosphären Druck leicht erfolgt. Die so erhaltenen, sehr wirksamen Lösungen geben stets die schöne Rosareaction mit Jod und nachherigem Ammoniakzusatz, was nicht mehr 
oder nur schwach der Fall ist, wenn ein höherer Druck von 8-12 Atmosphären ohne Zusatz von Schwefelsäure angewandt wird. Es entsteht dann bei dieser Probe eine grüne Färbung.

\section{Die unwirksame Modification des Epinephrins und die Ursache der Unwirksamkeit.}

Wenn zu der von Benzoesäure befreiten Lösung der gespaltenen Benzoylverbindung sehr verdünntes Ammoniak tropfenweise zugesetzt wird, so entsteht ein voluminöser flockiger Niederschlag von Epinephrin, welcher Anfangs farblos ist, aber bald eine dunkle Farbe annimmt, weshalb man ihn rasch auf dem Saugfilter mit kaltem Wasser, dann etwas kaltem absoluten Alkohol (der jedoch etwas davon löst), dann mit Aether nachwäscht und unter Verreiben im warmen Achatmörser rasch trocknet. Das Epinephrin bildet so ein schwach graues Pulver, fast unlöslich in Wasser, aber leicht löslich in verdünnten Mineralsäuren, Eisessig und Essigsäureanhydrid, und zeigt alle Reactionen des ursprünglichen Epinephrins mit Ausnahme der empfindlichen Rosafärbung mit Jodwasser und Ammoniak. Ich habe dieses getrocknete freie Epinephrin früher für ganz wirkungslos gehalten, allein weitere Versuche mit grösseren Mengen haben indes doch eine schwache Wirkung noch erkennen lassen. So lange die erwähnte Rosafärbung noch mit sehr geringen Mengen erhalten wird, ist auch noch die intensive volle physiologische Wirksamkeit vorhanden. Dieselbe wird aber nicht nur aufgehoben durch Spaltung bei zu hoher Temperatur, sondern auch, wie erwähnt, wenn die Base aus ihren Salzen befreit und getrocknet wird. Ein wirksames Salz büsst aber bei der direkten Umwandlung in ein anderes Salz nichts von seiner Wirksamkeit ein; so habe ich das Sulfat aus der Lösung des Pikrats in Essigäther dargestellt, ohne dass die Wirksamkeit verloren gegangen wäre. ${ }^{1}$ ) Wenn man ferner

1) So habe ich auch das salzsaure, schwefelsaure und benzoesaure Salz ineinander und in das Pikrat übergeführt, ohne dass Verlust der Wirksamkeit zu beobachten war. Wenn aber das Sulfat auf $110^{\circ} \mathrm{C}$. vier Stunden lang erhitzt wird, hat es seine Wirksamkeit zum grössten Theile verloren. Ein solches Präparat brachte erst in 23 facher Dosis die normale Wirkung hervor. 
die Base aus den Salzen fällt, rasch wäscht und sofort wieder in verdïnnten Säuren löst, ist noch eine gewisse beträchtliche Wirksamkeit vorhanden, aber, je länger man die freie Base sich selbst überlässt, je mehr man mit ihr manipulirt, desto mehr nimmt ihre Wirksamkeit ab. Es erinnert dieses Verhalten sehr an die Labilität und leichte Veränderlichkeit des esoAmidoacetophenons, Amidoäthylaldehyds und des Diamidoacetons, welche, aus ihren Salzen befreit, sich sehr bald umlagern.

\section{Analytische Resultate.}

\section{Epinephrin, $\mathrm{C}_{17} \mathrm{H}_{15} \mathrm{NO}_{4}$.}

Wie schon hervorgehoben, habe ich früher aschenhaltiges Epinephrin unter den Händen gehabt. Wenn man aber von dem Pikrat ausgeht, erhält man leicht aschenfreie Produkte. Einige Analysen habe ich bereits im Johns Hopkins Hospital, Bulletin für September-October 1898, mitgetheilt. Die Resultate waren folgende:

$0,145 \mathrm{~g}$ Substanz, in vacuo über Schwefelsäure bei $100^{\circ} \mathrm{C}$. getrocknet, gaben $0,3675 \mathrm{~g} \mathrm{CO}_{2}$ und $0,0684 \mathrm{~g} \mathrm{H}_{2} \mathrm{O}$, oder $69,12 \% \mathrm{C}$. und $5,24 \% \mathrm{H}$. Eine zweite Verbrennung von $0,1862 \mathrm{~g}$ Substanz, aus einer anderen und etwas modificirten Darstellung herrührend und wie das eben beschriebene Präparat getrocknet, gab $0,473 \mathrm{~g} \mathrm{CO}_{2}$ und $0,0102 \mathrm{~g}$ $\mathrm{H}_{2} \mathrm{O}$, oder $69,28 \% \mathrm{C}$ und $6,09 \% \mathrm{H}$. Eine Stickstoff-Bestimmung nach Dumas mit Substanz aus der eben genannten Darstellung ergab folgendes Resultat: $0,1784 \mathrm{~g}$ Substanz gaben $7,8 \mathrm{ccm}$. $\mathrm{N}$ bei $21^{\circ} \mathrm{C}$. und bei einem Barometerstand von $761 \mathrm{~mm}$. Daher $\mathrm{N}=5 \%$.

\section{Gefunden}

$$
\begin{aligned}
& \mathrm{C}=69,12 \% \quad 69,28 \% \\
& \mathrm{H}=5,24 \% \quad 6,09 \% \\
& \mathrm{~N}=\quad \tilde{5}, 00 \%
\end{aligned}
$$

Berechnet für $\mathrm{C}_{17} \mathrm{H}_{15} \mathrm{NO}_{4}$

$$
\begin{aligned}
& \mathrm{C}=68,68 \% \\
& \mathrm{H}=5,05 \% \\
& \mathrm{~N}=4,71 \% .
\end{aligned}
$$

Epinephrinpikrat, $\mathrm{C}_{17} \mathrm{H}_{15} \mathrm{NO}_{4} \cdot \mathrm{C}_{6} \mathrm{H}_{2}\left(\mathrm{NO}_{3}\right)_{3} \mathrm{OH}$.

Pikrinsäure schlägt das Epinephrin aus seinen Lösungen in verdünnten Mineralsäuren zum grössten Theile nieder. Die Filtrate geben stets noch die Vulpian'sche Reaction. mit Eisenchlorid ziemlich intensiv. Die Herstellung des Pikrats geschah in folgender Weise: Das aus der Lösung des gespaltenen Benzoats mit sehr verdünntem Ammoniak gefällte freie Epinephrin 
wurde nach dem Waschen wieder in warmer verdünnter Schwefelsäure von $2 \%$ gelöst und hierauf durch Zusatz von Natriumpikrat das Pikrat der Base ausgefällt. So erhalten, bildet es eine gelbliche oder bräunliche, harzige Masse. Aus sehr verdünnten Lösungen erhalten, zeigt es eine Tendenz, sich in Büscheln prismatischer Krystalle auszuscheiden. Nach Waschen mit kaltem Wasser, worin es nur wenig löslich ist, wird es bei $50^{\circ} \mathrm{C}$. getrocknet, in Essigäther gelöst (worin es leicht löslich ist) und aus der Lösung durch das $5-6$ fache Volumen Aethyläther ausgefällt. Das Produkt bildet so ein gelbes, sandiges Pulver, das aus minutiösen Sphärnkrystallen besteht. Wenn Aether nur bis zum Eintritt einer Trübung zugesetzt wird, so scheiden sich allmählich an der Wandung der Gefässe sphärische Aggregate wenig gut ausgebildeter Krystalle ab, welche die Grösse eines Stecknadelkopfes besitzen und durch leichten Druck in fächerförmige Fragmente mit radiären Streifen zerfallen. Das Salz ist leicht löslich in Alkohol, Essigäther, Chloroform, Methylal, Acetal und Amylenhydrat, unlöslich oder nur sehr wenig löslich in Aethyläther, Ligroin und Benzol.

Die Versuche, durch Umkrystallisiren aus Wasser zu besser ausgebildeten Krystallen zu gelangen, schlugen fehl; das Produkt war theils amorph, theils in minutiösen Sphärokrystallen wieder ausgefallen. Auf Platinblech erhitzt, verpufft es und brennt mit röthlicher russender Flamme. Mit feingepulvertem Kupferoxyd gemischt, brennt es ruhig ab. Nach wiederholter Fällung aus Essigäther stellt es ein hellgelbes Pulver dar, welches $\mathrm{zu}$ folgenden Analysen diente: 0,()492 g hinterliessen im Platintiegel keine Spur von Asche.

Bei der Elementaranalyse wurde ein ziemlich langes Kupferschiffchen verwendet und das Pikrat kalt mit dem Kupferoxyd gemischt (wegen seiner leichten Zersetzlichkeit), worauf wahrscheinlich der etwas zu hohe Wasserstoffgehalt zurückzuführen ist. Die Analysen gaben folgende Resultate:

$0,1806 \mathrm{~g}$ Substanz bei $110^{\circ} \mathrm{C}$. getrocknet gab $0,3496 \mathrm{~g} \mathrm{CO}_{2}$ und $0,0655 \mathrm{~g} \mathrm{H}_{2} \mathrm{O}$.

0,2926 g Substanz ebenfalls bei $110^{\circ}$ C. getrocknet gab $27 \mathrm{ccm}$. $\mathrm{N}$ bei $20,5^{\circ}$ C. und unter einem Barometerdruck von $760,2 \mathrm{~mm}$. gemessen. 


$\begin{array}{cc}\text { Gefunden } & \text { Berechnet für } \mathrm{C}_{15} \mathrm{H}_{15} \mathrm{NO}_{4} \cdot \mathrm{C}_{6} \mathrm{H}_{2}\left(\mathrm{NO}_{2}\right)_{3} \mathrm{OH} \\ \mathrm{C}=52,79 \% & \mathrm{C}=52,47 \% \\ \mathrm{H}=4,03 \% & \mathrm{H}=3,42 \% \\ \mathrm{~N}=10,54 \% & \mathrm{~N}=10,65 \% .\end{array}$

Ein nicht ganz reines Produkt habe ich erhalten, als ich das am Boden des sich im Autoclaven befindenden Gefässes nach der Spaltung noch vorgefundene harzige Produkt mit warmer 1\% iger Schwefelsäure behandelte, die so gewonnene Lösung mit verdünntem Ammoniak fällte und nach dem Waschen rasch wieder in 2\% iger Schwefelsäure löste und mit Natriumpikrat fällte. Nach Lösen in Essigäther und Fällen mit Aethyläther diente das Produkt zur Analyse.

$0,2098 \mathrm{~g}$ bei $110^{\circ} \mathrm{C}$. getrocknet lieferten $0,4230 \mathrm{~g} \mathrm{CO}_{2}$ und $0,0768 \mathrm{~g} \mathrm{H}_{2} \mathrm{O}$, entsprechend:

$$
\begin{aligned}
& \mathrm{C}=54,98 \% \\
& \mathrm{H}=4,06 \%
\end{aligned}
$$

\section{Epinephrinbisulfat, $\mathrm{C}_{17} \mathrm{H}_{15} \mathrm{NO}_{4} \cdot \mathrm{H}_{2} \mathrm{SO}_{4}$.}

Zur Darstellung diente das in beschriebener Weise gereinigte Pikrat, welches in concentrirter Lösung in Essigäther mit absolutem Alkohol, dem etwas verdünnte Schwefelsäure von $25 \%$ zugesetzt war, versetzt wurde. Hierbei fällt das Sulfat aus, das im Ueberschuss des sauren Alkohols sich indessen wieder löst. Nun wird eine grosse Menge Aether zugefügl, wodurch das Sulfat als körniges, grauweisses Pulver ausfällt. Wenn jedoch zu viel einer alkoholischen Schwefelsäure angewandt wird, so wird die Ausscheidung durch einen Wassergehalt zähe und ist dann schwerer zu behandeln. Nach Waschen mit Aether, dem etwas absoluter Alkohol zugesetzt wird, löst man es in warmem Alkohol von 95-97\% und fällt nochmals mit Aether. Dem Alkohol setzt man behufs rascherer Lösung eine Spur Schwefelsäure zu. Das Produkt ist ziemlich schwer löslich in Wasser, doch empfiehlt es sich nicht, es aus Wasser umzukrystallisiren, da es beim Behandeln mit kochendem Wasser, dem etwas Schwefelsäure zugesetzt ist, sich partiell zersetzt. Wenn man einige Cubikcentimeter des weiter unten beschriebenen wirksamen Sulfats bei mässiger Temperatur einengt und die concentrirte Lösung einige Zeit im Exsiccator 
sich selbst überlässt, findet man nach etwa einem Tag die Seiten und den Boden des Gefässes mit einem Netzwerk kleiner hübscher Prismen überzogen. Dieses Salz ist nur mässig beständig, denn nach einigen Wochen bildet sich in der Lösung ein körriger Bodensatz. erhalten:

Bei $100^{\circ}$ C. im Vacuum getrocknet, wurden folgende Zahlen

$$
\begin{array}{cc}
0,0903 \mathrm{~g} \text { lieferten } 0,0533 \mathrm{~g} \mathrm{BaSO}_{4} . \\
\text { Gefunden } & \text { Berechnet für } \mathrm{C}_{17} \mathrm{H}_{15} \mathrm{NO}_{4} \cdot \mathrm{H}_{2} \mathrm{SO}_{4} \\
\mathrm{H}_{2} \mathrm{SO}_{4}=24,80 \% . & \mathrm{H}_{2} \mathrm{SO}_{4}=24,81 \% .
\end{array}
$$

Obwohl die bisher beschriebenen Salze wirkungslos waren, da die Bereitungsweise aus der freien Base Umlagerungen nicht ausschliesst, so haben diese Analysen doch wenigstens die Aufstellung der empirischen Formel ermöglicht.

\section{Herstellung physiologisch wirksamer Verbindungen des Epinephrins.}

Wie oben schon auseinander gesetzt, werden bei der Spaltung der Benzoylverbindung bei Gegenwart von etwas Schwefelsäure unter einem Druck von 3-5 Atmosphären im Autoclaven äusserst wirksame Lösungen von Epinephrin erhalten, welche noch die rosarothe Färbung mit Jodwasser und verdünntem Ammoniak in ausgezeichneter Weise liefern. Nach Entfernung der Benzoesäure wird das Epinephrin direkt als Pikrat ausgefällt durch Zusatz von Natriumpikrat. Wir umgehen somit hier die Isolirung der freien Base, ein Verfahren, welches, wie oben erwähnt, zu inactiven Produkten führen würde. Wenn wir die Herstellung des Niederschlags fractionirt vornehmen, werden die zuletzt erhaltenen Antheile mit einer stickstoffreichen Substanz verunreinigt erhalten. So wurde in einem Fall im ersten Antheil 13,02\% N, in der dritten Fraction aber $16,66^{0} \% \mathrm{~N}$ gefunden.

Auch die physiologischen Versuche zeigten durch den Einfluss auf den Blutdruck, dass eine verunreinigende Substanz vorhanden ist, besonders in den letzten Fractionen. Bei der Injection dieser letzteren findet man, das auf das Eintreten des 
Steigens des Blutdruckes bald ein deutliches Sinken folgt, was bei den reinen Salzen niemals beobachtet wird.

Die weitere Reinigung des wirksamen Pikrats geschah durch wiederholtes Lösen in Essigäther und Fällen mit Aethyläther, ferner durch Ueherführen in andere Salze.

\section{Ueber die physiologische Wirksamkeit des Pikrats.}

Um zu zeigen, wie wirksam das rohe Pikrat ist, will ich die folgenden Versuche erwähnen. Nachdem das rohe Pikrat mit kaltem Wasser gewaschen und in einem Strome $50^{\circ}$ C. warmer Luft getrocknet wurde, löste ich es in Essigäther und fällte mit Aethyläther. Hierauf wurde eine Lösung in $45^{\circ} \%$ igem Alkohol von solcher Stärke hergestellt, dass jeder Cubiccentimeter $3,4 \mathrm{mg}$ des Pikrats enthielt. Bei der Injection von $0,35 \mathrm{ccm}$. dieser Lösung, enthaltend $0,0011 \mathrm{~g}$ des Pikrats, in die Jugularvene eines $6850 \mathrm{~g}$ wiegenden Hundes mit durchschnittenen Vagi, stieg der Blutdruck sofort von 102 auf $148 \mathrm{~mm}$. Quecksilber. Bei einem zweiten Versuch, bei welchem $0,0042 \mathrm{~g}$ des rohen Pikrats in die Jugularvene eines $6850 \mathrm{~g}$ wiegenden Hundes mit durchschnittenen Vagi injicirt wurden, stieg der Blutdruck von 122 auf $210 \mathrm{~mm}$. Quecksilber. Bei diesem Versuche wurde die alkoholische Lösung des Pikrats vor der Injection mit Wasser soweit verdünnt, dass sie nur noch 15\% Alkohol enthielt. Hierbei wurde etwas Pikrat in Form einer Trübung ausgefällt. Das so injicirte Pikrat führte zu folgendem bemerkenswerthen Resultat: Der Blutdruck blieb ungefähr zehn Minuten lang auf dem hohen Stancle und erst nach fünfzehn Minuten war der Druck wieder normal geworden, aber niemals wurde hier das geringste Anzeichen eines weiteren Sinkens unter den normalen Druck wahrgenommen. Wie wir später sehen werden, ist aber hier keineswegs noch die volle Wirkung des Epinephrins hervorgetreten, denn die leichter in Wasser löslichen Salze wie das Bisulfat und speciell das Hydrochlorat entwickeln eine ganz überraschende Fähigkeit, schon in sehr kleinen Dosen den Blutdruck zu erhöhen. 
Analytische Daten für das wirksame rohe Pikrat.

$0,1618 \mathrm{~g}$ des erst injicirten Pikrats, im Vacuumexsiccator bis zur Gewichtsconstanz getrocknet, lieferten $0,2974 \mathrm{~g} \mathrm{CO}_{2}$ und $0,0585 \mathrm{~g}$ $\mathrm{H}_{2} \mathrm{O}$, entsprechend: $\mathrm{C}=50,13 \%$

$$
\mathrm{H}=4,02 \% \text {. }
$$

$0,1840 \mathrm{~g}$ lieferten $23,8 \mathrm{ccm} . \mathrm{N}$ bei $16,5^{\circ}$ C. und unter einem Barometerdruck von $769,2 \mathrm{~mm}$. gemessen, entsprechend: $\mathrm{N}=15,22 \%$.

In einem zweiten Fall wurde das rohe Pikrat nicht erst durch fractionirte Fällung gereinigt, sondern direkt in das Bisulfat verwandelt, und aus diesem wurde dann wieder das Pikrat hergestellt.

$0,1863 \mathrm{~g}$ dieses Pikrats lieferten $0,3787 \mathrm{~g} \mathrm{CO}_{2}$ und 0,0635 g $\mathrm{H}_{2} \mathrm{O}$, entsprechend: $\mathrm{C}=55,43 \%$

$$
\mathrm{H}=3,78 \% \text {. }
$$

$0,1720 \mathrm{~g}$ lieferten $20 \mathrm{ccm}$. $\mathrm{N}$ bei $25,50 \quad$ C. und unter einem Barometerdruck von $76 \check{\mathrm{mm}}$. gemessen, entsprechend: $\mathrm{N}=13,02 \%$.

Auch in diesem Falle hat sich das Pikrat als höchst wirksam erwiesen. Aus den mitgetheilten Analysen folgt, dass das wirksame Pikrat noch mit verschiedenen Substanzen verunreinigt ist. Um nun noch reinere Produkte $\mathrm{zu}$ erhalten, habe ich bei meinen neuesten Versuchen, um den Prozentsatz der fremden Benzoate $\mathrm{zu}$ vermeiden, nur sehr verdünnte Extracte der Drüsen verwendet. Es ist ferner klar, dass beim Ausgehen von einem unreinen Pikrat behufs Darstellung anderer Salze die meisten Verunreinigungen entfernt werden können. Ich habe deshalb den Versuch aufgegeben, das active Pikrat als solches weiter zu reinigen, sondern habe meine Aufmerksamkeit auf die Herstellung anderer activer Salze aus dem Pikrat gerichtet, wozu das Pikrat vorzüglich geeignet ist. Hierbei müssen wässerige Lösungen vermieden werden.

\section{Wirksames Bisulfat.}

Das active Bisulfat wird in ziemlicher Reinheit in der folgenden Weise erhalten. Man löst das Pikrat in Essigäther, versetzt mit alkoholischer Schwefelsäure und fällt es fractionirt mit dem 4-6 fachen Volumen Aethyläther. Nach gründlichem Waschen mit einer Mischung von Alkohol und Aether wird diese erste Fraction in warmem Alkohol von $95 \%$ gelöst, 
filtrirt und das Filtrat mit Aether wieder gefällt, wobei man wieder nur die erste und grössere Fraction in Betracht zieht. Man operirt dabei so rasch, dass die Absorption von Wasser aus der Alkohol-Aetherlösung seitens des Niederschlags vermieden wird. Es wird dann ein staubartiges, aschefreies, fast weisses und in hẹissem Wasser ziemlich lösliches Pulver erhalten, welches physiologisch sehr wirksam ist und sich in trockenem Zustand beliebige Zeit aufbewahren lässt.

Die Analysen dieses Salzes gaben folgende Resultate:

$0,0888 \mathrm{~g}$ im Platintiegel verbrannt lieferten keine Spur von Asche. Der Tiegel hatte dasselbe Gewicht wie zuvor.

Beim Erhitzen blähte sich das Salz, entwickelte widrigriechende Gase und hinterliess eine Menge von mässig schwer verbrennlichem Kohlenstoff. In seinem Verhalten bei der Verbrennung im Tiegel erinnert das Epinephrin sehr an Alkaloide wie das Morphin. 0,2390 g im Vacuumexsiccator getrocknet und im Bleichromatrohre mit vorgelegter reducirter Kupferspirale verbrannt, lieferten $0,4548 \mathrm{~g} \mathrm{CO}_{2}$ und $0,1124 \mathrm{~g}$ $\mathrm{H}_{2} \mathrm{O}$, entsprechend: $\mathrm{C}=51,89 \%, \mathrm{H}=5,23 \%, 0,2278 \mathrm{~g}$ im gewöhnlichen Luftbad während 5 Stunden bei $110^{\circ}$ G. getrocknet und wie eben beschrieben verbrannt, lieferten $0,4426 \mathrm{~g} \mathrm{CO}_{2}$ und $0,1038 \mathrm{~g} \mathrm{H}_{2} \mathrm{O}$, entsprechend $\mathrm{C}=52,98 \mathrm{H}=5,06$.

$0,1800 \mathrm{~g}$ des Salzes, bei $110^{\circ}$ getrocknet, lieferten nach Liebig $0,0970 \mathrm{~g} \mathrm{BaSO}_{4}$, entsprechend $22,66 \%$ Schwefelsäure.
Gefunden,
Gefunden,
Berechnet für im Vacuum getrocknet bei $110^{\circ} \mathrm{C}$. getrocknet I II

$$
\begin{array}{rrr}
\mathrm{C}=51,89 \%, 0 & \mathrm{C}=52,98 \% & \mathrm{C}=51,64 \% \\
\mathrm{H}=5,23 \% \% & \mathrm{H}=5,06 \%, & \mathrm{H}=4,30 \% \\
\mathrm{H}_{2} \mathrm{SO}_{4}= & 22,66 \% & \mathrm{H}_{2} \mathrm{SO}_{4}=24,81 \% .
\end{array}
$$

Es geht hieraus hervor, dass dieses wirksame Salz noch nicht ganz rein ist; denn die Zahlen für $\mathrm{C}$ und $\mathrm{H}$ sind etwas zu hoch, besonders bei der bei $110^{\circ} \mathrm{C}$. getrockneten Probe, was auf einen schädigenden Einfluss dieser hohen Temperatur deuten könnte. Die Schwefelsäurebestimmung der ebenfalls bei dieser Temperatur getrockneten Probe gab auch eine $\mathrm{zu}$ niedrige Zahl. Immerhin ist die Abweichung nur gering und die früher aus der freien Base abgeleitete Formel kann als bestätigt gelten. Ich werde weiterhin zeigen, dass diese Formel sich aus manchen Derivaten wie die Acetylverbindung ergibt.

Die zweite Fraction des Sulfats, erhalten von späteren 
Fractionen des Pikrats, enthält mehr Kohlenstoff und weniger Schwefelsäure als das eben beschriebene Salz. Sie wurde in Alkohol gelöst, dem eine Spur Schwefelsäure zugesetzt war, und mit Aether gefällt. Im Vacuum bei $110^{\circ} \mathrm{C}$. getrocknet, lieferte es $56,22 \% \mathrm{C}, 5,20 \% \mathrm{H}$ und $22,29 \% \quad \mathrm{H}_{2} \mathrm{SO}_{4}$. Die Existenz eines neutralen Sulfats muss wohl ebenfalls in Betracht gezogen werden, welches $\mathrm{C}=58,95 \%, \mathrm{H}=4,61$ und $\mathrm{H}_{2} \mathrm{SO}_{4}=14,17 \%$ verlangen würde. Es ist recht wohl möglich, dass unter veränderten Bedingungen, besonders bei anderem Wasser und Schwefelsäuregehalt der Lösungen, ein Gemisch beider Sulfate niedergeschlagen werden könnte.

Es wurde nur ein Versuch gemacht, das neutrale Sulfat $\left(\mathrm{C}_{17} \mathrm{H}_{15} \mathrm{NO}_{4}\right)_{2} \mathrm{H}_{2} \mathrm{SO}_{4}$ darzustellen. Es wurden hierbei die späteren Fractionen von schwefelsäurearmen Lösungen, denen ein grosser Ueberschuss von Aether zugesetzt war. Das Material reichte leider zu einer vollständigen Analyse nicht aus, aber die Schwefelsäurebestimmung spricht zu Gunsten meiner Ansicht. 0,1584 g dieses Sulfats lieferte $0,057 \mathrm{~g} \mathrm{BaSO}_{4}=15,13 \%$ Schwefelsäure, während die Formel $\left(\mathrm{C}_{17} \mathrm{H}_{15} \mathrm{NO}_{4}\right)_{2} \mathrm{H}_{2} \mathrm{SO}_{4}$, wie eben angegeben, $\mathrm{H}_{2} \mathrm{SO}_{4}=14,17 \%$ verlangt.

\section{Epinephrin-Hydrochlorat und -Hydrobromat.}

Ausserordentlich leicht lösliche Salze sind das chlor- und bromwasserstoffsaure Salz, erhalten aus der Lösung des Pikrats in Essigaether durch Versetzen mit alkoholischer Lösung von $\mathrm{HCl}$ resp. HBr. (Das HJ-Salz wurde noch nicht darzustellen versucht.) Diese Salze sind grau bis braun, je nach dem $\mathrm{Zu}$ stande der Reinheit. Sie werden gereinigt durch Wiederlösen in absolutem Alkohol und Auffangen des Filtrats in viel Aether. Die aloholischen Lösungen färben sich rasch dunkel. Wegen ihrer leichten Löslichkeit dürften sie besonders zu therapeutischen Zwecken geeignet sein. Eine Probe des HBr-Salzes, aus einer dritten Fraction des Pikrats hergestellt, wurde im Vacuum getrocknet und mit dem folgenden Resultat analysirt: $0,2149 \mathrm{~g}$ gaben $0,1029 \mathrm{~g} \mathrm{HBr}=20,62 \% \mathrm{HBr}$.

Verlangt für $\mathrm{C}_{17} \mathrm{H}_{15} \mathrm{NO}_{4}$. $\mathrm{HBr}: 21,42 \% \mathrm{HBr}$.

Das Hydrochlorat wurde für physiologische und chemische 
Versuche verwendet, welche weiter unten beschrieben werden. Eine kleine Menge diente zur Herstellung des Chloroplatinats, welches aber sehr rasch dunkelt und sich verändert, so dass die Analyse nicht verlässlich ist. Nochmals in heissem Alkohol gelöst und in viel Aether filtrirt, fiel es in braunen Flocken aus, welche nach Waschen mit Alkoholäther und schliesslich mit Aether analysirt wurden. Der flockige Niederschlag enthielt $10,13 \% \mathrm{Pt}$.

\section{Ueber Triacetylepinephrin.}

Diese Verbindung bildete sich bei dreistündigem Kochen von Epinephrinbisulfat $(0,7 \mathrm{~g})$ mit einem Ueberschuss von Essigsäureanhydrid und wasserfreiem Natriumacetat am Rückflusskühler. Bei nachheriger Behandlung mit Wasser schied sich die Triacetylverbindung als harzige Masse aus. Diese wurde nach dem Waschen in Chloroform gelöst und das Filtrat in Petroleumäther aufgefangen, wobei sich das Produkt in Flocken und Körnchen ausschied, welche Reinigungsmethode nochmals wiederholt wird. Es bildet nun ein fast weisses, nicht krystallinisches, staubartiges Pulver. Die Ausbeute betrug von obengenannter Menge Bisulfat $=0,34 \mathrm{~g}$. Der bei der Wasserbehandlung des Reactionsproduktes in Lösung gebliebene Antheil kann durch Ausschütteln mit Chloroform ganz gewonnen werden.

\section{Analyse.}

$0,1612 \mathrm{~g}$ bei $80^{\circ} \mathrm{C}$. über $\mathrm{H}_{2} \mathrm{SO}_{4}$, im Vacuum getrocknet, lieferten $0,3829 \mathrm{~g} \mathrm{CO}_{2}$ und $0,069: 3 \mathrm{~g} \mathrm{H}_{2} \mathrm{O}$.

$$
\begin{array}{cc}
\text { Gefunden } & \text { Berechnet für } \mathrm{C}_{17} \mathrm{H}_{12} \mathrm{NO}_{4}\left(\mathrm{CO} . \mathrm{CH}_{3}\right)_{3} \\
\mathrm{C}=64,78 \% & \mathrm{C}=65,24 \% \\
\mathrm{H}=4,81 \% & \mathrm{H}=4,96 \%
\end{array}
$$

Ich stellte nun eine grössere Menge des Acetylderivats dar und zwar diesmal aus einem Bisulfat, welches aus dem mit Essigäther aus der ursprünglichen Pikratmutterlauge ausgeschüttelten Pikrat erhalten worden war. $3,83 \mathrm{~g}$ dieses Bisulfats lieferten bei der Wasserbehandlung nach der Acetylirung als harzige Fällung $=1,14 \mathrm{~g}$ des Triacetylprodukts und ferner noch 2,99 g beim Ausschütteln dieser Mutterlaugen mit Chloro- 
form. $\mathrm{Zu}$ den Mutterlaugen wurden aber die Mutterlaugen aus der ersten, sowie noch jene aus einer zweiten Probedarstellung hinzugefügt. Aus Obigem ist ersichtlich, dass die Ausbeute des Acetylderivats eine sehr gute ist.

- Eine vollständige Analyse wurde von dem erwähnten, mit Chloroform ausgeschüttelten Produkt ausgeführt.

$0,1628 \mathrm{~g}$, im Vacuumexsiccator getrocknet, lieferten $0,3823 \mathrm{~g} \mathrm{CO}_{2}$ und $0,0766 \mathrm{~g} \mathrm{H}_{2} \mathrm{O}$, entsprechend $\mathrm{C}=64,04 \%$ und $\mathrm{H}=5,22 \%$.

$0,36 \tilde{2}^{2} \mathrm{~g}$, in gleicher Weise getrocknet, lieferten nach Dumas $12,8 \mathrm{ccm}$. N bei $21, \tilde{0}^{\circ} \mathrm{C}$ und unter einem Barometerdruck von $762 \mathrm{~mm}$. gemessen, entsprechend $\mathrm{N}=3,99 \%$.

$0,2985 \mathrm{~g}$ bei $100^{\circ}$ C., im Vacuum über Schwefelsäure getrocknet, lieferten $9,5 \mathrm{ccm}$. $\mathrm{N}$. bei $21,5^{\circ} \mathrm{C}$ und bei einem Barometerstand von $762 \mathrm{~mm}$. gemessen, entsprechend $\mathrm{N}=3,62 \%$.

$0,2835 \mathrm{~g}$, im Vaccumexsiccator während mehrerer Wochen getrocknet, lieferten nach der Verseifung mittelst der weiter unten beschriebenen Methoden 0,12053 g Essigsäure, entśprechend 0,0864 g Acetyl $=30,47 \%$ Acetyl.

$0,3631 \mathrm{~g}$, bei $100^{\circ}$ in Vacuum über Schwefelsäure getrocknet, lieferten, nach der gleichen Methode verseift, 0,1524 $\mathrm{g}$ Essigsäure, entsprechend 0,10922 g Acetyl $=30,08 \%$ Acetyl.

$$
\begin{aligned}
& \text { Gefunden für das } \\
& \text { ausgefüllte } \\
& \text { Acetylprodukt } \\
& \text { Gefunden für das mit Chloroform } \\
& \begin{array}{l}
\mathrm{C}=64,78 \% \\
\mathrm{H}=4,81 \%
\end{array} \\
& \mathrm{C}=64,04 \% \\
& \mathrm{H}=5,22 \% \\
& \mathrm{~N}=3,62 \% \text { (bei } 100^{\wedge} \text { ('. getrocknet) } \\
& \mathrm{N}=3,99 \% \\
& \mathrm{C}_{2} \mathrm{H}_{3} \mathrm{O}=30,4 \% \% \\
& \mathrm{C}_{2} \mathrm{H}_{3} \mathrm{O}=30,08 \% \text { (bei } 100^{\circ} \mathrm{C} \text {. getrocknet). } \\
& \begin{array}{l}
\text { Berechnet für } \\
\mathrm{C}_{17} \mathrm{H}_{12} \mathrm{NO}_{4}\left(\mathrm{CO} . \mathrm{C}_{3}\right)_{3}
\end{array} \\
& \mathrm{C}=65,24 \% \\
& \mathrm{H}=4,96 \% \\
& \mathrm{~N}=3,31 \% \\
& \mathrm{C}_{2} \mathrm{H}_{3} \mathrm{O}=30,50 \%
\end{aligned}
$$

Aus den Analysen ist ersichtlich, dass das mit Chloroform ausgeschüttelte Produkt für Kohlenstoff etwas zu niedrige Zahlen aufweist. Das diesem entsprechende ausgefällte Acetylderivat war leider bei zu hoher Temperatur getrocknet und gab bei der Analyse $\mathrm{C}=63,45^{\circ} \%, \mathrm{H}=5,06$. Das Minus an Kohlenstoff ist bei diesem Antheil unzweifelhaft auf eine durch das Trocknen herbeigeführte geringe Zersetzung ${ }^{1}$ ) zurückzuführen.

1) Im Gehalt an Stickstoff, Wasserstoff und Acetyl sind die durch zu scharfes Trocknen verursachten Schwankungen geringer, aber immerhin noch bemerkbar. 
Folgende Resullate sollen diese Meinung erhärten. Während eine bei gewöhnlicher Temperatur getrocknete Probe des mit Chloroform ausgeschüttelten Produktes, wie oben angegeben, $64,04 \% \mathrm{C}$ und $5,22 \% \mathrm{H}$ lieferte, gab dieses Produkt bei $100^{\circ}$ 6-8 Stunden erwärmt (wobei Bräunung eintritt) 62,62\% $61,55 \% \mathrm{C}$ und $4,91-5,18 \% \mathrm{H}$. Gewichtsconstanz wird zwar bei $100^{\circ}$ erhalten, aber, wie erwähnt, erst nach einer gewissen Veränderung. Beim Trocknen sollte $80^{\circ}$ nicht überschritten werden.

Das Triacetylderivat ist bis jetzt das am reinsten erhaltene active Derivat. Es wird, wie erwähnt, aus der Benzoylverbindung, welche zuerst in das Pikrat und dann in das Bisulfat verwandelt wird, erhalten. Erst dann, wenn durch diese Umwandlungen Unreinigkeiten entfernt sind, schreitet man zur Darstellung der Triacetylverbindung. Ob wir daraus sicher auf drei Hydroxylgruppen schliessen dürfen, müssen weitere Studien über die in dem Epinephrin enthaltenen Atomgruppen entscheiden.

\section{Ueber das für Bestimmung der Acetylgruppen eingeschlagene Verfahren.}

Die Acetylverbindung wird leicht durch verdünnte Alkalien angegriffen. Ich wandte eine 5\% \%ige Lösung von Aetznatron an, womit ich die Verbindung zuerst gelinde bis zur Lösung erwärmte und dann noch 15 Minuten kochte. Die Mischung nimmt dabei eine dunkelbraune Farbe und einen an Coniin und Piperidin erinnernden Geruch an, genau wie bei den Salzen des Epinephrins, wenn sie in alkalischer Lösung erwärmt werden. Die Mischung wurde nach dem Erkalten mit Phosphorsäure ${ }^{1}$ ) versetzt und in üblicher Weise vorsichtig destillirt. Die Vorlage enthielt $18-20 \mathrm{ccm}$. einer ca. $\frac{\mathrm{N}}{5}$ Kalilösung. Der Kolben wurde zuletzt in ein Oelbad von 160 bis $180^{\circ}$ C. gesetzt, dann nochmals nach Zusatz von etwas

1) Bei dem Phosphorsäurezusatz entsteht ein dunkelbrauner, melaninartiger Niederschlag, auf den ich später zurückkommen werde. 
Wasser erhitzt, was dreimal wiederholt.wurde, um die letzten Spuren Essigsäure überzudestilliren.

Die Kalilösung in der Vorlage wurde nun mit Schwefelsäure titrirt, von der $1 \mathrm{ccm} .=0,01618 \mathrm{BaSO}_{4}=0,00833$ Essigsäure entsprach, unter Benutzung von Lakmus als Indicator. Die schwach sauer reagirende Flüssigkeit wurde schliesslich am Rückflusskühler gekocht, bis alle aus dem Aetznatron oder der organischen Substanz stammende $\mathrm{CO}_{2}$ entfernt war, und mit der titrirten Kalilösung $(1 \mathrm{ccm} .=0,01313 \mathrm{~g}$. Essigsäure) zurücktitrirt. Selbstverständlich wurden noch Kontrollversuche ausgeführt, welche die absolute Reinheit der Reagentien darthaten. Nur etwas $\mathrm{CO}_{2}$ wurde erhalten. Weitere Versuche mit $0,15 \mathrm{~g}$ Epinephrinbisulfat bewiesen mir, dass bei der Behandlung von Epinephrin selbst mit Natronlauge und Phosphorsäure keine flüchtige, organische Säure, sondern nur etwas $\mathrm{CO}_{2}$ entsteht. Ferner habe ich mich überzeugt, dass kein Verlust beim Kochen von verdünnter Essigsäure am Rückflusskühler entsteht.

Ist das Epinephrin, wie v. Fürth annimmt, ein Hydrodioxypyridin?

Es mag hier am Platze sein, das von 0 . v. F ürth ${ }^{1}$ ) aus dem blutdrucksteigernden Körper der Nebenniere erhaltene Acetylderivat einer Betrachtung zu unterziehen. Die zweite Abhandlung dieses Autors wurde ungefähr um dieselbe Zeit veröffentlicht, als meine zweile Abhandlung erschien, und ich hatte bis jetzt keine Gelegenheit, auf jene Resultate zurückzukommen. Jenes Acetylprodukt war die einzige Verbindung, welche v. Fürth in Händen hatte, und dieselbe war erhalten worden durch Acetylirung von Produkten, welche entweder vorher einer Reduction mit Zink und Schwefelsäure unterworfen oder aus dem Organ durch verdünnte Zinksulfatlösung extrahirt waren. Keine zwei Proben des Acetylprodukts, aus verschiedenen Mengen der Drüse stammend, stimmten in ihrer Elementarzusammensetzung überein. Der C-Gehalt schwankte von $51,53-59,70 \%$, der H-Gehalt von $5,18-6,58 \%$ und der

1) Diese Zeitschr., Bd. XXVI, S. 1 . 
Stickstoff von 4,80-6,20\%. Aus dem gewonnenen Mittel berechnet v. Fürth, dass das von ihm analysirte Produkt das Triacetylderivat des Tetrahydrodioxypyridins oder Dihydrodioxypyridins, $\mathrm{C}_{5} \mathrm{H}_{9} \mathrm{NO}_{2}$ oder $\mathrm{C}_{5} \mathrm{H}_{7} \mathrm{NO}_{2}$, ist.

Die analytischen Resultate welche mir, nach mehrfachen reinigenden Proceduren des Epinephrins, die Acetylverbindung lieferte, sind weit verschieden von denen des Herrn v. Fürth, der C-Gehalt ist höher und der N-Gehalt niedriger als v. Fürth annimmt. Der grosse Unterschied unserer Resultate ergibt sich schon aus den verschiedenen Mengen Essigsäure, welche aus dem Acetylprodukt abgespalten wurden. So fand v. Fürth 64,43-69,62\% Essigsäure, während ich 42,51 bis $43,11 \%$ fand. Bei Annahme von v. Fürth's Formeln würden 74,69 oder 75,31\% Essigsäure erfordert werden, während meine Formel $42,55 \%$ verlangt, also weit besser mit der Beobachtung stimmt. Es kann kaum einem Zweifel unterliegen, dass das von v. Fürth analysirte Produkt ein Gemenge von Epinephrin mit andern Substanzen war. Nur die Isolirung einer Anzahl von Salzen und andern Derivaten kann hier die Sicherheit der Reinheit geben und zu einer zuverlässigen Formel führèn.

\section{Phenylhydrazin und Epinephrin.}

Ich habe vor Kurzem beobachtet, dass die Acetylverbindung des Epinephrins bei entsprechender Behandlung mit Phenylhydrazinacetat ein Phenylhydrazinderivat liefert. Das Produkt besitzt noch basische Eigenschaften und lässt sich als Pikrat ausfällen, in ein Sulfat u. s. w. verwandeln. Aus dem Sulfat fällt bei genauer Neutralisation mit verdünntem Ammoniak ein flockiges Epinephrinhydrazon aus, welches sich aber in seinen Eigenschaften scharf von Epinephrin selbst unterscheidet. So gibt es eine hübsche Rosafärbung bei Zusatz von Jodwasser und Ammoniak, aber diese verschwindet nicht beim Kochen, sondern tritt noch besser hervor. Ferner erfolgt beim Kochen mit schwachen Alkalien (bis $\mathrm{zu} 25 \% \mathrm{NaOH}$ ) nicht eine braune oder braun-schwarze Färbung, sondern eine schöne Rosafärbung, welche bei stärkeren Lösungen in eine tiefe blau-violette Färbung 
übergeht und welche längere Zeit bestehen bleibt. Concentrirte $\mathrm{H}_{2} \mathrm{SO}_{4}$ löst es mit carminrother Farbe auf, concentrirte $\mathrm{HCl}$ mit weinrother Farbe. Die Reaction mit $\mathrm{H}_{2} \mathrm{SO}_{4}+\mathrm{KMnO}_{4}$ bleibt erhalten und die mit dem Mandelin'schen Reagens zeigt das Stadium der blassrothen Färbung in deutlicherer Weise als das Epinephrin selbst. Fehling's Lösung wird beim Stehen mit der Verbindung nicht reducirt, wohl aber beim Kochen nach vorheriger Spaltung des Hydrazons mit Alkalien oder Säuren.

In diesem Phenylhydrazinderivat sind die Acetylgruppen, wie es scheint, nicht mehr enthalten. Ich habe jedoch Gründe für die Annahme, dass auch bei Beibehaltung der Acetylgruppen ein Hydrazon zu erhalten ist.

Es unterliegt also kaum einem Zweifel, dass eines der Sauerstoffatome des Epinephrins den Charakter eines Aldehyds oder Ketonsauerstoffs besitzt. Ich hoffe, später Weiteres mittheilen zu können, wenn die vermuthete Keton- respective Aldehydnatur durch weitere Gruppenreactionen begründet worden ist.

Vergleichende physiologische Versuche mit Proben wirksamen Bisulfats, dargestellt aus den Filtraten von den mit Natriumpikrat erhaltenen Niederschlägen.

Ich habe oben bereits mitgetheilt, dass nach Zersetzung des Benzoylprodukts und Entfernung der Benzoesäure Natriumpikrat im Ueberschuss zwar Epinephrinpikrat fällt, aber doch noch erheblich Epinephrin gelöst bleibt, denn das Filtrat gibt noch starke Reactionen auf die Base und ist physiologisch noch äusserst wirksam. Essigäther entzieht beim Schütteln das wirksame Pikrat der Lösung. Das hieraus dann dargestellte Bisulfat stimmt mit dem früher beschriebenen, aus dem direkt gefällten Pikrat dargestellten völlig überein, ja ich schloss aus der übergrossen Wirksamkeit, dass jenes Sulfat noch reiner ist als dieses.

Dieses veranlasste mich, jenes Bisulfat dazu zu verwenden, den Minimumbetrag zu finden, der den Blutdruck noch $\mathrm{zu}$ steigern im Stande sei. Diese Versuche sind folgende: Von 
einer Probe dieses Bisulfats reichten 0,00013 g hin, den Blutdruck um $14 \mathrm{~mm}$. Hg zu erhöhen bei einem kleinen Hunde mit durchschnittenen Vagi. Auch von einer zweiten Probe reichte eine sehr geringe Dosis hin, doch hinderte ein Unfall die genauere Bestimmung. Von einem dritten Präparat waren nur $0,00011 \mathrm{~g}$ nöthig, den arteriellen Blutdruck um $16 \mathrm{~mm}$. $\mathrm{zu}$ erhöhen bei einem $6,08 \mathrm{~kg}$. wiegenden Hunde. Bei einer Injection von $0,00043 \mathrm{~g}$ dieses Präparats stieg der Blutdruck unmittelbar um $60 \mathrm{~mm}$. Es ist somit klar, dass noch eine entschiedene physiologische Wirkung von einer so geringen Menge als $0,000018 \mathrm{~g}$ pro Kilo Körpergewicht vom Bisulfat hervorgerufen werden kann, entsprechend $0,000013 \mathrm{~g}$ der freien Base. Bei Injection des 5-6fachen Betrags erfolgte eine sehr bedeutende und langanhaltende Blutdrucksteigerung. Bei diesen Versuchen wurde mit einer genau calibrirten Spritze in die Jugularvene injicirt; die benutzten Lösungen enthielten $0,001 \mathrm{~g}$ pro Cubikcentimeter.

Da die verschiedenen Proben des eben beschriebenen, aus den Filtraten von der Natriumpikratfällung gewonnenen Bisulfats nicht völlig in Bezug auf den Schwefelsäuregehalt stimmten, dürfte wohl in einem oder anderen Falle noch eine geringe Verunreinigung enthalten sein. Auch ist der Schwefelsäuregehalt dieses sehr wirksamen Salzes immer noch etwas niedriger gefunden worden, als von meiner Formel verlangt wird. Die erhaltenen Resultate waren wie folgt:

$0,177 \mathrm{~g}$ des im ersten der eben beschriebenen physiologischen Versuche benutzten Bisulfats wurden in verdünnter, heisser Salzsäure gelöst und die Schwefelsäure direkt mit $\mathrm{BaCl}_{2}$ ausgefällt: $\mathrm{BaSO}_{4}$ erhalten $=0,0935 \mathrm{~g}$, entsprechend $\mathrm{H}_{2} \mathrm{SO}_{4}=22,21 \%$.

$0,0838 \mathrm{~g}$ des im zweiten physiologischen Versuche angewandten Präparats lieferten $\left.0,04.56 \mathrm{~g} \mathrm{BaSO}{ }_{4}, 1\right)$ entsprechend $22,88 \% \mathrm{H}_{2} \mathrm{SO}_{4}$.

$0,2402 \mathrm{~g}$ des im dritten physiologischen Versuche angewandten Präparats lieferten $0,1347 \mathrm{~g} \mathrm{BaSO}_{4}$, entsprechend $\mathrm{H}_{2} \mathrm{SO}_{4}=23,58 \%$.

$0,1824 \mathrm{~g}$ dieses Präparats, bei $110^{\circ} \mathrm{C}$. im Trockenschrank getrocknet, lieferten $0,3205 \mathrm{~g} \mathrm{CO} \mathrm{CO}_{2}$ und $0,0815 \mathrm{~g} \mathrm{H}_{2} \mathrm{O}$.

1) Diese und die folgende Analyse wurden nach Liebig ausgeführt, weil das Bisulfat, wie alle Salze des Epinephrins, mit der Zeit an Löslichkeit einbüsst. 


$\begin{array}{ccc}\text { Gefunden } & \text { Verlangt für } & \text { Verlangt für } \\ & \mathrm{C}_{17} \mathrm{H}_{15} \mathrm{NO}_{4} \cdot \mathrm{H}_{2} \mathrm{SO}_{4} & \left(\mathrm{C}_{17} \mathrm{H}_{15} \mathrm{NO}_{4} \cdot \mathrm{H}_{2} \mathrm{SO}_{4}\right)+11_{2} \mathrm{H}_{2} \mathrm{O} \\ \mathrm{C}=47,92 \% & \mathrm{C}=51,64 \% & \mathrm{C}==48,34 \% \\ \mathrm{H}=4,90 \% & \mathrm{H}=4,30 \% & \mathrm{H}=4,74 \% \%\end{array}$

Manches spricht dafür, dass dieses das reinste sämmtlicher dargestellter Salze ist und dass wir anzunehmen haben, dass unter anderem das wirksame sich von dem unwirksamen Präparat dadurch unterscheidet, dass jenes ein, respectiv ein und einhalb Molekül Wasser mehr enthält. Bei dieser Annahme $\left(1^{1 / 2} \mathrm{H}_{2} \mathrm{O}\right.$ mehr) würde das reine Bisulfat $48,34 \% \mathrm{C}$, $4,74 \% \mathrm{H}$ und $23,22 \% \mathrm{H}_{2} \mathrm{SO}_{4}$ verlangen. Diese Vermuthung würde durch die analytischen Resultate des Acetylproduktes keine Widerlegung erfahren, da ja bei der Acetylverbindung das supponirte Wasser eingebüsst werden könnte. Hierbei mag auch auf die Thatsache hingedeutet werden, dass man auf dreierlei verschiedenen Wegen ein Bisulfat darzustellen vermag, welches immer genau die nämlichen Reactionen zeigt und eine sehr annähernd gleiche Zusammensetzung aufweist. Auch führt das Bisulfat, auf welche Weise es auch dargestellt wird, anscheinend immer zu dem gleichen Acetylprodukt.

\section{Farben-Alkaloidreactionen der Epinephrinsalze.}

Beide, die Salze der wirksamen und der unwirksamen Modification des Epinephrins, verhalten sich charakteristisch gegen die gewöhnlichen, durch Erzeugung von Färbungen ausgezeichneten Alkaloidreagentien. Die feinste Reaction ist die des Mandelin'schen Reagens, welches selbst bei leisen Spuren von Epinephrin dunkelstahlblaue Streifen hervorruft, an denen ein rasches und feines Farbenspiel von rasch verschwindendem Violett zu Kirschroth und Rosa stattfindet. Wenn jenes Reagens mit 5-6 Theilen $\mathrm{H}_{2} \mathrm{O}$ verdünnt angewendet wird, bringt es beim Eintröpfeln in eine Epinephrinsalz-Lösung eine schöne violette Färbung hervor, die bald blassroth wird und dann sogleich verschwindet, bei jedem Tropfen der in die Lösung fällt.

Concentrirte Schwefelsäure löst Epinephrin und seine Salze auf und ruft eine oliven- bis schmutzig braun-grüne Färbung hervor. Wird in jene Lösung ein Körnchen $\mathrm{KMnO}_{4}$ 
gegeben, so entsteht eine schöne bläulich-violette Färbung, welche bald einen mehr indigoblauen Ton annimmt und lange unverändert fort besteht. Von der ähnlichen Reaction beim Strychnin unterscheidet sie sich dadurch, dass die Reihe der intermediären Uebergänge nicht beobachtet wird. $\mathrm{H}_{2} \mathrm{SO}_{4}$ mit $\mathrm{MnO}_{2}$ oder $\mathrm{H}_{2} \mathrm{SO}_{4}$ mit $\mathrm{K}_{2} \mathrm{Cr}_{2} \mathrm{O}_{7}$ geben ein ähnliches rasches, aber weniger deutliches Farbenspiel, wie Mandelin's Reagens. Fröhde's Reagens, $\mathrm{H}_{2} \mathrm{SO}_{4}+$ Rohrzucker, concentrirte oder rauchende $\mathrm{HNO}_{3}$ geben keine farbige Reaction.

\section{Verhalten zu alkaloidfällenden Reagentien.}

$\mathrm{Zu}$ diesen Versuchen dienten lediglich die wirksamen Salze, welche eine eminent reducirende Kraft (an die der Amidoaldehyde erinnernd) besitzen, was dadurch illustrirt wird, dass aus Jodsäure Jod, aus ammoniakalischer Silberlösung $\mathrm{Ag}$ abgeschieden wird und K-Ferricyanid $\mathrm{zu}$ K-Ferrocyanid reducirt wird; auch $\mathrm{PtCl}_{4}$ und $\mathrm{AuCl}_{3}$. werden reducirt, wenn auch langsamer. Fehling's Lösung liefert keine Abscheidung von $\mathrm{Cu}_{2} \mathrm{O}$.

Die in der folgenden Tabelle beschriebenen Niederschläge wurden meistens in verdünnten Lösungen erhalten, welche in ihrer Concentration, schätzungsweise, zwischen $1 / 100$ bis $1 / 500$ schwankten.

\begin{tabular}{|c|c|}
\hline Reagentien & Eigenschaften des Niederschlags \\
\hline Jodjodkalium & Brauner, flockiger Niederschlag, Amorph. \\
\hline Kaliumcadmiumjodid & Weisser, \\
\hline Kaliumquecksilberjodid & 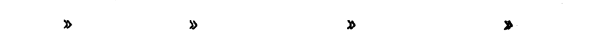 \\
\hline $\begin{array}{l}\text { Jodchlorid und Jod- } \\
\text { trichlorid }\end{array}$ & $\begin{array}{l}\text { Brauner oder gelblich brauner, flockiger, } \\
\text { bald sich zu einem käsigen Niederschlag } \\
\text { umformend. Amorph. }\end{array}$ \\
\hline Kupferacetat & $\begin{array}{l}\text { Grünlich-weisser, flockiger Niederschlag, } \\
\text { Amorph. }\end{array}$ \\
\hline Pikrinsäure & $\begin{array}{l}\text { Gelber, flockiger Niederschlag. Fällung nicht } \\
\text { vollständig. }\end{array}$ \\
\hline $\begin{array}{l}\text { Concentrirte Lösung Sulfo- } \\
\text { cyanammonium }\end{array}$ & $\begin{array}{l}\text { Weisser, flockiger Niederschlag, löst sich } \\
\text { auf Zusatz von Wasser. Amorph. }\end{array}$ \\
\hline $\begin{array}{c}\text { Concentrirte Zinkchlorid- } \\
\text { Lösung }\end{array}$ & Weisser flockiger Niederschlag. Amorph. \\
\hline
\end{tabular}




\begin{tabular}{|c|c|}
\hline Reagentien & Eigenschaften des Niederschlags \\
\hline $\begin{array}{l}\text { Platinchlorid ( } 10 \% \text { ige } \\
\text { wässerige Lösung) }\end{array}$ & $\begin{array}{l}\text { Gelber, flockiger, amorpher Niederschlag, in } \\
\text { Alkohol löslich, wird rasch dunkel gefärbt, } \\
\text { reducirt sich beim Stehen, unter Aus- } \\
\text { scheidung von Pt. }\end{array}$ \\
\hline $\begin{array}{l}\text { Goldchlorid ( } 10 \% \text { ige } \\
\text { wässerige Lösung) }\end{array}$ & $\begin{array}{l}\text { Verhält sich wie der eben beschriebene } \\
\text { Niederschlag. }\end{array}$ \\
\hline Phosphormolybdänsäure & Weisser. flockiger, amorpher Niederschlag. \\
\hline Phosphorwolframsäure & $»$ \\
\hline Gerbsäure & $\begin{array}{l}\text { Weisser, flockiger, amorpher Niederschlag } \\
\text { aus concentrirten Lösungen. }\end{array}$ \\
\hline $\begin{array}{l}\text { Quecksilberchlorid (gesätt. } \\
\text { wässerige Lösung) }\end{array}$ & Kein Niederschlag aus verdünnten Lösungen. \\
\hline Quecksilbercyanid (idem) & $»$ \\
\hline $\begin{array}{c}\text { Kaliumbichromat (gesätt. } \\
\text { Lösung) }\end{array}$ & Brauner, flockiger, amorpher Niederschlag. \\
\hline Kaliumferrocyanid (idem) & Weisser, \\
\hline $\begin{array}{l}\text { Einleiten von Chlor oder } \\
\text { Brom }\end{array}$ & $\begin{array}{l}\text { Flockiger, bald käsig werdender Nieder- } \\
\text { schlag. }\end{array}$ \\
\hline
\end{tabular}

\section{Ueber die Zersetzungsprodukte des Epinephrins bei Behandlung mit Alkalien.}

W'ir haben bereits die leichte Veränderlichkeit des Epinephrins unter verschiedenen Einflüssen erwiesen. Diese ist sogar noch bedeutender, als es nach meinen ersten Versuchen den Anschein hatte. Wenn wir in Betracht ziehen, welche geringe Mengen hinreichen, die physiologische Reaction auf den Blutdruck zu erzengen, dürfen wir uns aber nicht darüber wundern, dass nach schädigender Einwirkung einer $5 \%$ igen Natriumhydratlösung ${ }^{1}$ ) auf wirksame Salze eine auf diese Weise noch auffindbare Menge der Veränderung entging.

Meine weiteren Studien über die wirksamen Salze haben mich ferner überzeugt, dass die carminrothe Substanz, welche aus den wirksamen Salzen unter Einfluss von sehr verdünntem Alkali und gelinder Oxydation (Jodwasser und $\mathrm{NH}_{3}$ ) mit dem Epinephrin in inniger Beziehung steht, und nicht etwa bloss

1) Johns Hopkins Hospital Bulletin, July 1897. 
einer Verunreinigung zuzuschreiben ist, wie ich in meiner ersten Abhandlung vermuthungsweise äusserte. Dieser rothe Körper nimmt bei Anwesenheit stärkerer Alkalien sehr rasch eine braune oder schwarze Färbung an. Diesen braunen Farbstoff habe ich näher untersucht.

Das beim Verseifen des Benzoylderivats im Autoclaven ohne Schwefelsäurezusatz erhaltene Produkt unterliegt bald einer Oxydation und nach einigen $\mathrm{W}^{\mathrm{T}}$ ochen ist ein beträchtliches Sediment eines braunschwarzen Pigments gebildet. Lösungen des Epinephrins in organischen Säuren verhalten sich ebenso. Das Filtrat gibt dann weder chemische, noch physiologische Reactionen des Epinephrins. Eisenchlorid gibt dann keine grüne Färbung mehr, der Blutdruck wird nicht mehr erhöht.

Ein scheinbar damit identisches Produkt wird erhalten, wenn immer Lösungen des Epinephrins mit Alkaliüberschuss gekocht und mit Mineral- oder Essigsäure gefällt werden. Bei der Bestimmung der Acetylgruppen haben wir diese Beobachtung bereits gemacht und oben mitgetheilt. Das Pigment ist leicht in verdïnnten Alkalien löslich und hat im allgemeinen Verhalten manche Aehnlichkeiten mit den von verschiedenen Forschern aus thierischen Organen isolirten Melaninen. Wegen seiner sauren Natur nenne ich das Produkt Epinephrinsäure. Wenn es durch Behandeln des salzsauren Epinephrins mit Natronlauge von $2 \breve{5} \%$ gebildet und mit Schwefelsäure daraus niedergeschlagen wird, erhält es nach Waschen und Trocknen noch immer Stickstoff. Mit der Analyse bin ich noch beschäftigt, Mittheilung der Resultate wird folgen. Soweit bis jetzt ersichtlich ist, ist dieser Körper das Hauptumwandlungsprodukt.

Ein zweites Zersetzungsprodukt unter dem Einfluss von Alkalien ist von basischem Charakter. Eine mit Alkali übersättigte Lösung eines Epinephrinsalzes entwickelt schon bei gewöhnlicher Temperatur einen Geruch, der an den eines Gemisches von Coniin und Piperidin erinnert. Die Base, deren Dämpfe stark alkalisch auf Lakmus reagiren, kann zum kleinen Theil mit Aether ausgeschüttelt werden. Diese ätherische Lösung gibt Nebel mit HCl-Gas und mit Salzsäure sofort einen Niederschlag eines HCl-Salzes. Beim Verdunsten der mit HCl 
versetzten ätherischen Lösung hinterbleibt das HCl-Salz der Base in Form weisser harziger Kügelchen. Dieses Salz, in Wasser gelöst, gibt auf Zusatz von Alkali eine Ausscheidung von öligen Tropfen von dem ursprünglichen Geruch.

Jodkaliumquecksilber, Jodkaliumeadmium, Phosphorwolframsäure, Phosphormolybdänsäure und Tannin geben alle weisse flockige Niederschläge mit dem HCl-Salz. Platinchlorid gibt einen gelben käsigen Niederschlag, während Jodjodkalium rothbraune Flocken liefert. Andere Alkaloidreagentien wurden noch nicht versucht. Beim Schmelzen mit Zinkstaub entwickelt das HCl-Salz einen starken Pyrrolgeruch und die Fichtenspahnreaction wird in ausgezeichneter Weise erhalten.

$\mathrm{Ob}$ das basische Produkt einheitlich ist, oder aus mehreren Basen besteht, ist noch nicht entschieden; denn die Reindarstellung der basischen Bestandtheile ist mit Schwierigkeiten verbunden, indem schon die Destillation einen weiten zersetzenden Einfluss zu haben scheint. Ich zweifle deshalb, ob ich die basische Substanz frei von anderen Zersetzungsprodukten des Epinephrins erhalten habe. Soweit die Sache jetzt beurtheilt werden kann, sind die beiden Hauptprodukte der Epinephrinspaltung vermittelst Lösungen von Alkalien, eine Säure und eine (eventuell mehrere) Base, in welcher Beziehung das Epinephrin mit vielen Pflanzenalkaloiden übereinstimmt. Weitere Mittheilungen hoffe ich später machen zu können.

\section{Ueber die Zersetzungsprodukte des Epinephrins bei trockener Destillation und Destillation mit Zinkstaub.}

Versuche, über welche ich schon in meiner ersten $\mathrm{Ab}$ handlung berichtet habe, hatten bereits ergeben, dass das Epinephrin bei trockener Destillation unter Anderem Pyrrol und Amine liefert, dass es sich also in dieser Beziehung wie viele Alkaloide verhält. Beim Schmelzen des Epinephrins mit Zinkstaub in einem Strome trocknen Wasserstoffs und Auffangen der Produkte in einer Reihe kleiner Flaschen beobachtete ich ausser Aminen und Pyrrol eine Substanz vom Geruche des Benzaldehyds und ferner eine, deren Lösungen eine rosarothe Färbung an der Luft annahmen und weitere noch nicht 
näher studirte Produkte. Ob Pyridin unter diesen war, konnte ich nicht sicher darthun, obgleich ich einen Niederschlag mit Bromwasser und mit Kupfersulfat erhielt. Die schön blaue charakteristische Färbung tritt bei Anwendung von Kupfersulfat nicht hervor.

v. Fürth behauptet zwar beim Schmelzen seines wirksamen Materials und dessen Acetylverbindung bei Destillation mit Zinkstaub Pyridin erhalten zu haben und gibt ausser der Wahrnehmung eines ähnlichen Geruches noch Reactionen mit Jodkaliumquecksilber, Phosphorwolframsäure, Jodjodkalium, Sublimat und Platinchlorid als Gründe an. Er erwähnt aber nicht das Verhalten zu Bromwasser, Kupfersulfat, Eisen- und Cadmiumchlorid.

Meiner Ansicht nach hat v. Fürth den vollständigen Beweis für seine Angaben nicht geliefert, sondern nur dargethan, dass eine basische, flüchtige Substanz von pyridinähnlichem Geruch gebildet war. Ich bezweifle jedoch nicht, dass bei Wiederholung der Versuche mit reinem Material in grösserem Massstabe eine oder mehrere Basen der Pyrrol- oder Pyridinreihe gefunden werden, möglicher $\mathrm{K}^{\top}$ eise Pyridin selbst.

Ich habe schon darauf hingewiesen, dass, wenn ein Epinephrinsalz mit concentrirter alkoholischer Lösung von $\mathrm{KOH}+\mathrm{CHCl}_{3}$ behandelt wird, ein ekelhafter, an Carbylamine erinnernder Geruch wahrgenommnn wird. Aus allen hier erwähnten Versuchen ${ }^{1}$ ) will ich aber bis zur weiteren Erforschung vorläufig nur den Schluss ziehen, dass das Epinephrin eine labile Substanz ist, deren Hauptverhalten eine alkaloide Natur erweist.

\section{Skatol, ein Zersetzungsprodukt des Epinephrins.}

Von wesentlich höherem Interesse ist die Zersetzung, welche beim Schmelzen mit $\mathrm{KOH}$ vor sich geht, wobei der durchdringende Geruch des Skatols auftritt. Wenn die wässerige Lösung der Schmelze mit Aether ausgeschüttelt wird,

1) Ich habe noch nicht Gelegentreit gehabt, die eben beschriebenen Versuche mit meinem reinen neueren Präparate zu wiederholen. 
so hinterlässt der Aether beim Verdunsten kleine Tröpfchen von fäcalem Geruch, welche beim Auflösen in concentrirter $\mathrm{HCl}$ sofort die charakteristische rothe Färbung liefern, wie es Skatol bei sogar kleinen Mengen stets thut.

Eine alkoholische Lösung dieser Tröpfchen gibt mit einem mit $\mathrm{HCl}$ benetzten Fichtenspahn eine intensive rothe Färbung; eine Lösung in Benzol gibt mit einer Lösung von Pikrinsäure in Benzol unmittelbar einen Niederschlag von Pikrat in Form röthlicher Tröpfchen; ferner gibt die wässerige Lösung mit Schwefelsäure und Kaliumnitrit eine weisse Trübung, wie es auch Skatol thut. Auch Salkowski's Reaction wurde erhalten, wenn auch unvollkommen, denn die Erzeugung intensiver Färbungen würde grössere Mengen beansprucht haben, als mir zur Verfügung standen.

Es kann keinem Zweifel unterliegen, dass dieses Zersetzungsprodukt Skatol ist. Ich habe obige Versuche wiederholt durchgeführt mit kleinen Mengen des freien Epinephrins, seiner Salze und des spontan ausfallenden braunen Oxydationsprodukts. Dieses Verhalten gibt uns aufs Neue die Berechtigung, das Epinephrin den wahren Alkaloiden zuzuzählen. Nach Stöhr ${ }^{1}$ ).gibt auch Strychnin beim Erhitzen mit Kalk Skatol, ferner haben Hoffmann und Königs ${ }^{2}$ ) Indol aus Tetrahydrochinolin beim Durchleiten durch ein glühendes Rohr erhalten.

Aus den mitgetheilten Analysen geht hervor, dass die elementare Zusammensetzung des Epinephrins durch die Formel $\mathrm{C}_{17} \mathrm{H}_{15} \mathrm{NO}_{4}$ ausgedrückt werden kann. Die glatte Darstellung der Acetylverbindung spricht für einen Gehalt von drei Hydroxylgruppen, jedoch ist dieser Schluss nicht als ein endgültiger zu betrachten, da die Bindungsweise des Stickstoffs noch nicht festgestellt ist. Die vorläufigen Versuche mit Phenylhydrazin deuten auf das Vorhandensein einer Keton- resp. Aldehydgruppe hin. Schon jetzt wäre ein Einblick in die Constitution dieser interessanten Verbindung gewonnen, wenn wir annehmen dürften, dass das durch Schmelzen mit Kali entstehende Skatol

1) Berichte d. Deutsch. chem. Gesellsch., Bd. 20, S. 1108.

2) Ibid., Bd. XVI., S. 738. 
ein integraler Theil des Epinephrin-Moleküls sei und nicht etwa secundär durch beträchtliche Atomverschiebungen entstanden ist. Das brenzcatechinähnliche Verhalten des Epinephrins gegen Eisenchlorid spricht, wie schon von Fränkel¹) hervorgehoben, für eine benachbarte Stellung zweier der vorhandenen Hydroxylgruppen. Bei der Annahme, dass der Skatolrest als solcher im Molekül existirt, dürfte man sich die Zusammensetzung des Epinephrins in folgender Weise vorstellen:<smiles>CCCCCCC(=O)OCC(=O)OCC</smiles>

Eine solche Formel würde meiner schon früher ausgesprochenen Vermuthung, dass das Epinephrin eine Pyrrolbase ist, zu Recht bestehen lassen. Die ausgesprochene chromogene Natur des Epinephrins erführe dann auch eine Erklärung, sowie seine Entstehung im Thierkörper aus eiweissartigen Vorstufen. Eine nahe Beziehung $\mathrm{zu}$ der von $\mathrm{Nencki}^{2}$ ) aus faulendem Eiweiss isolirter Skatolessigsäure würde dann auch vermuthet werden dürfen.

Ich bin mir wohl bewusst, dass es noch viel Arbeit kosten wird, eine endgültige Constitutionsformel für das Epinephrin aufzustellen, und möchte obige Formel vorläufig nur als eine hypothetische, in einigem Maasse den Thatsachen entsprechende aufstellen. Sie soll unter Anderem meine Annahme in Bezug auf den Kerntypus des Epinephrins erläutern. Obwohl aus ihrer Betrachtung nicht ersichtlich ist, in welcher Weise die schon beschriebene Spaltung des Epinephrins in Epinephrinsäure und eine basische, coniinartige Substanz erfolgen soll, so ist doch zu erwähnen, dass auch diese Substanz beim Erhitzen mit Zinkstaub Pyrrol liefert. Ob Protokatechusäure oder eine mit ihr verwandte Substanz, aus Epinephrin abzuspalten ist, habe ich in letzter Zeit nicht $\mathrm{zu}$ entscheiden versucht. Jedoch

1) Wiener med. Blätter, 1896, Nr. 14-16.

2) Sitzungsber. d. k. Akad. d. Wissensch. in Wien, Bd. 98, Abth. II b; Mai 1889. 
habe ich schon vor zwei Jahren beobachtet, dass beim Erhitzen des damals gewonnenen Epinephrinbisulfats im zugeschmolzenen Rohre mit $25 \% \mathrm{HCl}$ auf $150^{\circ} \mathrm{C}$. eine geringe Menge einer in Aether löslichen Substanz erhalten wird, welche auf Zusatz von Eisenchlorid eine grüne Färbung annahm. Ich betrachtete diese Substanz damals als Epinephrin, welches der Einwirkung der Salzsäure entgangen war. Auch wurde mittelst Aether aus dem Röhreninhalt eine Säure gewonnen, welche sich nach Reinigen durch Sublimation der Benzoesäure ähnlich verhielt. v. F ̈̈rth ${ }^{1}$ ) hat auch gefunden, dass durch trockene Destillation eine Substanz erhalten wird, welche von Aether sowohl aus saurer als aus alkalischer Lösung aufgenommen wird und die Eisenreaction in gleicher Weise wie Brenzkatechin gibt. Es bestehen daher einige Thatsachen, welche die Anordnung des zweiten Benzolkerns in meiner vorläufigen Formel nicht als allzugewagt erscheinen lassen:

\section{Ueber die pharmakologische Wirkung des Epinephrins.}

Eine eingehende pharmakologische Prüfung des Epinephrins wurde noch nicht ausgeführt. Obgleich Extracte der getrockneten oder frischen Nebennieren sehr gut geeignet sind, die merkwürdige Einwirkung auf den Blutdruck und das Herz zu zeigen, sind sie doch zu ausführlicheren pharmakologischen Studien nicht geeignet, weil solche Extracte noch zu viele Verunreinigungen enthalten, worunter solche von beträchtlicher physiologischer Wirkung, wie daraus hervorgeht, dass eine Injection grösserer Mengen des aus den Handelsprodukten hergestellten wässerigen Extracts rasch den Tod herbeiführt, unter Symptomen, welche dem reinen Epinephrin nicht eigen sind. Meine Schüler haben solche Injectionen im vergangenen Winter öfters an Hunden und Kaninchen ausgeführt, um die den Blutdruck erniedrigende Wirkung des Chloroforms wieder aufzuheben. Es kam hierbei fünfmal vor, dass kurz nach Beginn des Ansteigens des Blutdrucks derselbe plötzlich wieder fiel, bis der Nullpunkt des Druckes erreicht war. Während des Fallens

1) Diese Zeitschr., Bd. XXIV, S. 150. 
war anfänglich die Athmung noch intakt, und beim Oeffnen des Brustkorbes ergab sich Stillstand ${ }^{1}$ ) des Herzens in Diastole. Wie ich zeigen werde, hat reines Epinephrinsalz in grösseren Mengen zunächst eine lähmende Wirkung und bleibt der Blutdruck noch einige Zeit auf hohem Stande, und es bedarf weiterer Injectionen, um bei Unterhaltung der künstlichen Athmung den Blutdruck fallen zu machen. Da es nun gar nicht möglich ist, dass die wässerigen Extracte bei den Versuchen meiner Schüler so viel Epinephrin enthielten, dass dadurch eine Lähmung des Herzens erfolgen konnte, bleibt nur der Schluss übrig, dass andere Constituenten der erwähnten Handelspräparate hierfür verantwortlich $\mathrm{zu}$ machen sind. Ich muss deshalb gegen intravenöse Injection zu grosser Dosen des wässerigen Nebennierenextracts bei Anästhesieunfällen meine warnende Stimme erheben. Auch sollten für den allgemeinen ärztlichen Gebrauch die Nebennieren mit grosser Sorgfalt präparirt werden.

\section{Lokale Wirkung der wirksamen Salze.}

Zahlreiche Forscher haben darauf aufmerksam gemacht, dass wässerige Nebennierenextracte bei der Conjuctiva und anderen Schleimhäuten eine Contraction der Blutgefässe herbeiführen. Diese Wirkung fand ich auch bei meinen wirksamen Epinephrinsalzen. Wenn die durch Reiben mit einem Glasstab oder einem Strom von Aetherdampf hyperämisch gemachte Conjunctiva eines Kaninchens mit etwas zerriebenem Epinephrinpikrat oder Bisulfat in Berührung kommt, so geht die Hyperämie sofort zurück. Dasselbe Resultat erfolgt nach Eintröpfeln einer Lösung eines leicht löslichen Epinephrinsalzes in den gerötheten Conjunctivalsack. Im ersten Falle bleibt die Wirkung sehr lange erhalten. Ein Tropfen einer starken Lösung des Hydrochlorats bringt auf der Zunge einen geringen Grad von Gefühllosigkeit und einen schwach bitteren Geschmack hervor.

1) Vgl. Oliver u. Schäfer, Journ. of Physiol., vol. 18, 1895, p. 238. Gottlieb, Arch. f. exp. Pathol. u. Pharmak., Bd. 38, S. 112. Gourfein, Compt. rend. Acad. d. sciences, C.XXI, 1895, 311. Joà u. Pellacani, Maly's Jahrb. d. Thier'chemie, XIII, 129. Marino-Zucco, ibid., XVIII, 231. 
Epinephrin ist ein Gift, welches das Athmungscentrum lähmt.

Versuch I.

Injection von $2 \mathrm{mg}$ des wirksamen Bisulfats in den dorsalen Lymphraum eines mittelgrossen Frosches hat Anfangs keine andere Wirkung, als die Athmungsfrequenz zu erhöhen. Nach 10 Minuten stellen sich Zeichen von Depression ein, denn das Thier nimmt nicht mehr seine normale Stellung ein, sondern liegt auf dem Bauche, obgleich es beim Reizen in der gewöhnlichen Weise springt.

\section{Versuch II.}

Eine Lösung von etwas weniger als $10 \mathrm{mg}$ Epinephrinhydrochlorid wurde in den dorsalen Lymphraum eines grossen kräftigen Frosches in zwei Dosen in einem Interval von $5 \mathrm{Mi}$ nuten injicirt. Vor der Injection war die Athemfrequenz 56 pro Minute, unmittelbar nach der ersten Injection stieg dieselbe auf 76 pro Minute. Während die zweite Injection gemacht wurde, fiel die Athemfrequenz auf 52, dann rasch auf 44 und wurde nun langsamer, bis 25 Minuten nach der ersten Injection die Athmung ganz stillstand. Während dieser Zeit war eine zunehmende Schwäche zu bemerken. 15 Minuten nach der ersten Injection machte das Thier beim Legen auf den Rücken nur schwache Gegenbewegungen, trotz der noch vorhandenen schwachen Athmung. Auf kräftige Reize reagirte es nur schwach. Nach Aufhören der Athmung konnte keine Spur mehr von Reflexwirkung hervorgebracht werden und alle willkürliche Bewegung hatte aufgehört. Bei Prüfung des freigelegten $\mathrm{N}$ ischiadicus mit einem schwachen secundären Strom, welcher bei einem Kontrollversuch an dem gleichnamigen Nerven eines enthirnten Frosches als wirksam gefunden wurde, wurde eine normale Muskelcontraction auf der Seite der Reizung herbeigeführt, was die Abwesenheit einer curareartigen Wirkung darthut.

Beim Blosslegen des Herzens wurde constatirt, dass dieses 58 Schläge pro Minute ausführte. Die Contractionen waren sehr vollständig, wie aus der Blässe des Ventrikels während 
jeder Systole hervorging. Die Herzwirkung erinnerte stark an die von Digitalis, wie auch schon von Gottlieb ${ }^{1}$ ) für das Herz des Warmblüters hervorgehoben worden ist.

Ein Kontrollfrosch zeigte nach Zerstörung seines Gehirns einen Herzschlag von 64 pro Minute.

Der Frosch dieses Versuches II blieb in einer feuchten Kammer und liess am folgenden Morgen keine Spur mehr des narkotischen Effects des Epinephrins erkennen. Er blieb nicht mehr auf dem Rücken liegen und beim Drücken des Fusses sprang er wie ein normaler Frosch. Aus diesen von mir noch einmal wiederholten Versuchen schliesse ich, dass das Epinephrin unter die Cerebro-Spinalgifte ${ }^{2}$ ) zu rechnen ist.

\section{Versuche an Kaninchen.}

Die bis jetzt an warmblütigen Thieren gemachten Versuche stimmen ganz mit den eben beschriebenen überein.

\section{Versuch I.}

5 ccm. einer Lösung des Epinephrinhydrochlorids, $25 \mathrm{mg}$ pro Cubikcentimeter enthaltend, wurden langsam in die Randvene des Ohrs eines Kaninchens von 1320 g injicirt. Die Injection des vierten Cubikcentimeters der Lösung war etwa halb vollendet, als das Thier, welches bis dato keinen Widerstand gezeigt hatte, plötzlich eine zuckende Bewegung machte. Vorher konnte nichts anderes als eine enorm beschleunigte Respiration und die heftige rasche Bewegung des Herzens beobachtet werden. Die Spritze wurde nun rasch zurückgezogen und das Thier auf den Boden gesetzt. Unmittelbar darauf traten heftige Krämpfe auf und nach wenigen Anfällen, die ganz an Blausäurewirkung erinnerten, hörte das Thier zu athmen auf.

1) Arch. f. exp. Pathol. u. Pharmak., Bd. 38, S. 104.

2) Die hier angeführten Versuche sollen nur einige der gröberen Wirkungen des Epinephrins darlegen. So sind bis jetzt von mir keine Versuche zur genaueren Erforschung seiner Wirkung auf das Rückenmark, Herz, Organe mit glatter Muskulatur u. s. w. ausgeführt worden. 


\section{Versuch II.}

Bei diesem sollte eine letale Dose in mehreren Abtheilungen und Intervallen einem Kaninchen beigebracht werden, um eine etwaige narkotische Wirkung beobachten zu können. Das Versuchsprotocoll ist folgendes:

Grosses, starkes männliches Kaninchen mit 140 Athemzügen pro Minute, erhält $10 \mathrm{mg}$ von Epinephrin-HCl, in eine Ohrvene. Unmittelbar darauf heftige Herzschläge und beschleunigte Athmung. Nach 3 Minuten 172 Athemzüge pro Minute, soweit Zählung möglich war, und nach 4 Minuten 184.

Nach 10 Minuten erfolgte eine weitere Injection von 34 mg. Athmung bald 152, darauf 140. Herz schlägt rasch und mit grosser Heftigkeit.

Nach weiteren 15 Minuten werden weitere $20 \mathrm{mg}$ injicirt. Wegen der eintretenden Contraction der Blutgefässe wurde es nun sehr schwierig, eine Ohrvene zu finden, die weit genug für die Injection war. Die Ohrgefässe erschienen nun, wenn das Ohr gegen das Licht gehalten wurde, als minutiöse rothe Striche. Da bei der vorigen Injection schon ein Theil der Lösung in das Gewebe um die Ohrvene gelangte, wurde der Rest der Lösung, enthaltend $25 \mathrm{mg}$, in die Bauchhöhle injicirt. Obgleich das Thier im Ganzen $89 \mathrm{mg}$ in getheilten Dosen erhalten hatte, zeigte es doch keine anderen deutlichen Symptome als die bereits erwähnten vasculären und respiratorischen. Es war noch fähig, herumzuspringen, und obgleich es mir schien, dass die Fähigkeit, sich zu bewegen, beschränkter war als sonst, war ich doch über diesen Punkt nicht ganz sicher. Am folgenden Morgen war das Thier wieder völlig normal und frass wie gewöhnlich. Es scheint daher, dass bei grossen Dosen das Athemcentrum im Kaninchen gelähmt wird, bevor eine narcotische Wirkung entwickelt werden kann.

\section{Versuch III.}

Um zu erfahren, ob beim Eintritt der Athmungslähmung die Circulation noch keine Störung zeigt, wurde ein Kaninchen $1820 \mathrm{~g}$ schwer in Aetheranästhesie gehalten, seine Trachea 
mit einer registrirenden Trommel verbunden und die linke Carotis mit einem Hg-Manometer in der üblichen Weise, während die rechte Jugularvene mit einer Injectionscanüle verbunden wurde. Um 4 Uhr 18 Min. 14 Sek. wurde begonnen mit einer Injection von $106 \mathrm{mg}$ des Epinephrin-HCl, gelöst in $4 \mathrm{ccm} . \mathrm{H}_{2} \mathrm{O}$, sie wurde vollendet um 4 Uhr 19 Min. 55 Sek.

Mit dem ersten Moment begann der Blutdruck zu steigen und die Athemzüge ${ }^{1}$ ) wurden weder mehr frequent noch verstärkt, aber sehr bald darauf, um 4 Uhr 19 Min. 30 Sek., zeigte die Trommel kaum merkliche Erhöhungen des Athemhebels und um 4 Uhr 19 Min. 36 Sek. zeigte sich darauf eine gerade Linie; das Thier athmete nicht mehr. Es war also Lähmung ${ }^{2}$ ) der Athmung eingetreten, lange bevor die Injection beendet war! Mit dem Moment dieser Lähmung fing der Blutdruck langsam zu fallen an. Um 4 Uhr 24 Min. 40 Sek. wurde künstliche Respiration eingeleitet und um $4 \mathrm{Uhr}$ 26 Min. 20 Sek. hatte der Blutdruck wieder seinen hohen Stand eingenommen. Der Druck, welcher vor der Injection von $64-68 \mathrm{~mm}$. $\mathrm{Hg}$ schwankte, blieb nun auf dem hohen Niveau von $140 \mathrm{~mm}$. und zwar bis 4 Uhr $41 \mathrm{Min}$., als die

1) Die Athemzüge waren ror der Injection bei Aethernarcose 14 pro 10 Sekunden während dem Ansteigen des Blutdruckes, und 12 Sekunden bevor Respirationsstillstand eintrat, betrugen sie noch 13 pro 10 Sekunden, waren aber nun sehr bedeutend abgeschwächt. Die Einstellung des Marey'schen Tambours mit einem grossen Luftraum verbunden, bei gleichzeitiger partieller Oeffnung der Trachealcanüle, war nicht geeignet, feinere Grade der Verstärkung oder Abschwächung der Respiration darzulegen.

2) Badano hat auch das Aufhören der Respiration constatirt bei Injection von Nebennierenextract. Citirt in der Münch. med. Woch. 1898, C. 475, aus dem Ber. der Med. Akad. zu Genua vom 7. Febr. 1898. Nach Sorona und Moroni wirkt das kalt gewonnene wässerige Extract der Drüse erst excitirend, dann lähmend auf die Herzaction und ebenso auf die Respiration. Citirt, ibid., S. 939, aus Riform. Med. 1898, p. 459.

Gourfein's Versuche mit alkoholischen, aus wässerigen Auszügen der Drüse dargestellten Extracten erwiesen auch bei Säugethieren eine immer zunehmende Dyspnoe. Bei Unterhaltung künstlicher Athmung konnte er die Lähmung des Herzens nach weiterer Injection bewirken. Compt. rend., Acad. d. sc. CXXI (1895), p. 311. 
künstliche Respiration auf einige Sekunden unterbrochen wurde. Der Blutdruck fiel hierbei sofort und da die Hoffnung auf Rückkehr normaler Athmungsthätigkeit aufgegeben werden musste, wurde noch einmal zur künstlichen Athmung geschritten, bevor das Herz $\mathrm{zu}$ schwach wurde für weitere Einflüsse.

Um 4 Uhr 52 Min. wurden weitere $50-60 \mathrm{mg}$ des Epinephrin-HCl rasch injicirt, wodurch rapides Sinken des hohen Druckes auf $46 \mathrm{~mm}$. verursacht wurde, welcher Stand etwa 10 Sekunden anhielt, worauf um 4 Uhr 53 Min. der Druck wieder auf $110 \mathrm{~mm}$. anstieg.

Um 4 Uhr 53 Min. 30 Sek. wurde eine weitere rasche Injection von 50-60 mg gemacht, mit dem gleichen Erfolg, dass zunächst ein Sinken eintrat, aber diesmal war die Dose letal für das Herz, denn trotz ausgiebiger künstlicher Athmung fiel der Druck weiter zur Grundlinie, der Puls war nicht mehr fühlbar und beim Oeffnen der Brusthöhle wurde der Ventrikel in schlaffer Diastole vorgefunden, während die Vorhöfe noch schwach sich contrahirten und ihre Schläge in Gruppen erfolgten.

Es ist deshalb evident, dass das Epinephrin in übermässigen Dosen schliesslich auch das Herz warmblütiger Thiere lähmen kann und nicht nur die Athmung. Es geht ferner aus den Versuchen hervor, dass die toxische Dose dieser eigenthümlichen Substanz sehr viel und zwar vielleicht hundertmal höher liegt, als die für einen therapeutischen oder physiologischen Effect nöthige Dosis.

\section{Allgemeine Betrachtungen.}

Dass die Nebenniere ein lebenswichtiges Organ ist, steht wohl heute nicht mehr in Zweifel. Dass dem blutdrucksteigernden Bestandtheil dieser Drüse, dem Epinephrin eine physiologische Bedeutung zukommt, wird von Vielen als bewiesen angesehen und ist mindestens sehr wahrscheinlich. Es ist aber zu frühzeitig, dogmatisch über die Unentbehrlichkeit des Epinephrins für den Körper zu reden, denn die Nebenniere mag vielleicht noch andere wichtigere Leistungen zu verrichten haben, als die, das Epinephrin zu liefern. Immerhin haben 
die Anhänger der «inneren Secretions-»Theorie eine Anzahl interessanter Beobachtungen zu Tage befördert. So hat Cybulski ${ }^{1}$ ) zuerst bewiesen, dass das aus der Nebenniere abfliessende Blut die blutdrucksteigernde Substanz enthält, während das aus anderen Organen stammende venöse Blut keine Spur dieses Körpers aufweist. Langlois ${ }^{2}$ ), Biedl ${ }^{3}$ ) und Dreyer ${ }^{4}$ ). haben diese Thatsache dann auch zur Genüge bestätigt. · Biedl's Versuche haben es ferner sehr wahrscheinlich gemacht, dass die Produktion des Epinephrins unter dem Einfluss des Nervensystems steht, dass die Nervi splanchnici nicht nur die Vásodilatatoren, sondern auch die Secretionsnerven für die Nebennieren in ihren Bahnen führen. Dreyer hat dann kürzlich festgestellt, dass dem wirklich so ist, dass das Epinephrin in erhöhtem Maasse in das venöse Blut ausgeschieden wird, wenn der N. splanchnicus unterhalb des Zwerchfells durch Inductionsströme gereizt wird, und dass diese erhöhte Secretion. unabhängig ist von der gleichzeitig in der Drüse durch die Reizung hervorgerufenen Gefässerweiterung. Es scheint also bewiesen zu sein, dass das Organ mehr oder weniger Epinephrin zu liefern im Stande ist, je nach der Thätigkeit seiner secretorischen Nerven.

Was sind nun die Beziehungen des Epinephrins zur Addison'schen Krankheit? Die erste Frage, welche sich hier aufdrängt, ist die: Besteht irgend ein chemischer Zusammenhang zwischen dem Epinephrin selbst oder seinen Vorstufen und dem. bekannten, bei der Bronzekrankheit in der Haut und Schleimhäuten abgelagerten Pigment?

Wie bekannt, hat Mühlmann ${ }^{5}$ ) diese Frage dahin beantwortet, dass das Pigment der bronzegefärbten Haut aus oxydirtem Brenzcatechin bestehe. "In der Nebenniere wird Brenzcatechin producirt, in geringen Dosen gelangt es in das Blut.

1) Anzeiger d. Akad. d. Wissensch. in Krakau, 4. März 1895, aus. Szymonowicz, Pflüger's Archiv, Bd. 64, S. 145̆, citirt.

2) Revue scientifique, 1897, p. 303.

3) Pflüger's Archiv, Bd. 67, S. 477.

4) Americ. Journ. of Physiol., vol. II, p. 203.

5) Loc. cit. 
und wird durch den Harn ausgeschieden. Die überschüssige Menge des gebildeten Brenzcatechins, welche, falls sie ins Blut gekommen wäre, giftig wirken könnte, wird durch die Thätigkeit der sympathischen Ganglienzellen der Nebenniere oder des Ganglion solare eliminirt. Ist es einmal zu einer Ansammlung von Brenzcatechin im Organismus gekommen, so haben wir die Addison'sche Krankheit vor uns: das Brenzcatechin pigmentirt die Haut und ruft alle jene giftigen Erscheinungen hervor, welche die Bronzekrankheit charakterisiren.» ${ }^{1}$ ) Diese Theorie ist aber eine ganz irrige, denn es ist kein Brenzcatechin in der Nebenniere vorhanden.

Diese Frage lässt sich vorläufig gar nicht entscheiden. Es müssen erst chemische Versuche behufs Isolirung des in der Haut bei der Bronzekrankheit abgelagerten Pigments angestellt werden. Erst wenn die chemische Untersuchung etwas Definitives über die Natur dieses Pigments zu Tage gebracht hat, kann man von einer. Beziehung oder Nicht-Beziehung dieses Pigments zu einem Nebennierenbestandtheil reden.

Vorläufig werden alle Vermuthungen über den Ursprung des in Frage stehenden Pigments durch folgende Umstände vereitelt. Erstens dadurch, dass ähnliche Hautpigmentirungen bei verschiedenen anderen pathologischen Zuständen auftreten, so bei der chronischen Arsenvergiftung, bei gewerblichen Schädigungen, bei tiefgreifenden Ernährungsstörungen u. s. w.; zweitens dadurch, dass bei schnell tödtlich verlaufenden Fällen dieser Krankheit die Bronzefärbung oft ganz ausbleibt. Betreffs dieses letztgenannten Umstandes mag auf die pathologischanatomischen Erhebungen Fenwicks ${ }^{2}$ ) aufmerksam gemacht werden, nach welchen die Thatsache hierbei massgebend sein soll, ob zuerst, oder mehr ausgesprochen, die Marksubstanz oder die corticale Substanz degenerirt ist. In Fällen, wo die Marksubstanz frühzeitig und total verkäst ist, sollen die allgemeinen Symptome der Krankheit in sehr schwerer Form auf-

1) Virchow's Archiv, Bd. 146, S. 267.

2) Transactions Path. Soc. London, vol. 33, p. 353. 
treten, während die Pigmentation ausbleibt; der Tod tritt ein (innerhalb 5 Monaten), bevor es zur Pigmentation kommt.

Nun ist allgemein bekannt, dass das Epinephrin nur in der Marksubstanz und nicht in der Corticalsubstanz gebildet wird. Auch haben Schäfer und Oliver ${ }^{1}$ ) gezeigt, dass keine Spur des Epinephrins aus einer Nebenniere, deren Marksubstanz total verkäst ist, extrahirt werden kann. Daher würden auch die oben genannten Fälle ihre Beweiskraft gegen die Annahme der Entstehung des Pigments aus dem Epinephrin verlieren, denn es wäre in jenen Fällen gleich von Anfang an kein Epinephrin vorhanden. Wie und warum aber, bei Vorhandensein des Epinephrins, dieses in den Bronzefarbstoff verwandelt werden würde, bliebe immer noch ein Räthsel. Andererseits könnte man die Frage aufwerfen, ob nicht das fragliche Pigment aus einer Vorstufe des Epinephrins entstehe, und ob die Bronzefärbung nur eintritt, wenn die Drüse unfähig wird, jene Substanz zu bilden. Bei dem jetzigen Stande unserer Kenntniss der hier obwaltenden Verhältnisse kann man es nicht sicher behaupten, dass irgend eine Beziehung zwischen dem Epinephrin und dem Pigment der Bronzekrankheit existirt.

Immerhin ist es eine sehr auffallende Thatsache, auf welche in dieser Abhandlung schon mehrmals hingedeutet worden ist, dass Lösungen des Epinephrins so ausserordentlich leicht, so schon beim blossen Stehen an der Luft, ein braunes Pigment ausscheiden, welches sich in vieler Beziehung wie ein thierisches Pigment verhält. Die Unlöslichkeit dieser Substanz in Blutserum, wie ich bestätigen kann, würde sie zur Ablagerung in pigmentführenden Zellen geeignet machen.

Wie bei dem Verhältniss zwischen Epinephrin und dem Pigment der Bronzekrankheit, so steht es auch bei dem Verhältniss zwischen jener Substanz und anderen Symptomen dieser Krankheit, wie z. B. die ausgesprochenen Schwächeerscheinungen, der Kopfschmerz, die Anfälle von Schwindel, die terminalen schweren nervösen Erscheinungen, die Verdauungsstörungen

1) Journ. of Physiol., vol. 18 (1895), p. 269. 
u. s. w. Ein ursächlicher Zusammenhang ist auch hier vorläufig nicht zu postuliren. Es lässt sich nicht übersehen, in welchem Maasse oder ob überhaupt diese Symptome durch gänzlichen Wegfall oder zu geringe Produktion der blutdrucksteigernden Substanz verursacht werden. Auch sind wir ganz im Dunkel über eine etwaige Giftigkeit jener Substanzen, welche als Vorstufen des Epinephrins functioniren. Wir haben gesehen, dass das Epinephrin selbst, in übergrossen Mengen in den Kreislauf gebracht, ein starkes Nervengift ist. Bei übermässiger Produktion, in Folge Reizzustände der Nebennieren, könnte also eine Vergiftung, eine "Autointoxication», erfolgen. Andererseits würde ebenfalls eine Vergiftung erfolgen können bei totaler Degeneration der Drüse, wenn die Vorstufen ${ }^{1}$ ) des Epinephrins diesem in ihrer Giftigkeit nur annähernd glichen.

Wir bewegen uns aber hier in einem Reiche der Speculation, und ich möchte es wohl verstanden wissen, dass meine obigen Bemerkungen nur als auf Möglichkeiten hindeutend aufzufassen sind. Es wäre unerlaubt, den Thatsachen der zukünftigen Forschung voraneilen zu wollen.

Eine andere Frage, welche Aussichten auf eine baldige Lösung aufweist, ist die die normale Ausscheidung des Epinephrins betreffende.

Ich habe den Urin eines Hundes untersucht, welcher beträchtliche Mengen eines wirksamen Epinephrinsalzes $(20 \mathrm{mg}$ oder mehr) in die Ingularvene injicirt enthalten hatte. 'In diesem Falle nahm der Urin auf Zusatz von Natronlauge eine dunkelbraune Farbe an, ferner eine grüne mit Eisenchlorid, eine Färbung, die noch besser hervortrat bei Filtration nach diesem Zusatz. Es ist deshalb klar, dass nach intravenöser Injection das Epinephrin zum Theil unverändert im Harne

1) Hierbei ist auch in Betracht zu nehmen, dass die Drüse noch andere giftige Substanzen als das Epinephrin enthält, so z. B. die stark blutdruckerniedrigende Substanz. Eine chemisch-physiologische Erklärungsweise des Zustandekommens der oben genannten Symptome müsste auch gegenüber allen solchen Körpern, beziehungsweise deren Vorstufen, gerecht werden. 
wieder erscheint, oder wenigstens nur so weit verändert wird, dass die Reaction mit Eisenchlorid noch erhalten wird. Ob ein solcher Urin blutdrucksteigernd wirken kann, habe ich noch nicht geprüft.

Es ergibt sich nun naturgemäss die Frage, wie das Epinephrin im normalen Zustande im Urin wieder erscheint. Die Vermuthung, dass es unter normalen Umständen völlig oxydirt wird, ist nach Obigem, sowie nach dem Kerntypus dieser Substanz kaum anzunehmen. Wir haben ferner gesehen, dass das Epinephrin durch Einwirkung verschiedener oxydirender Agentien intensive Färbungen annimmt, und deshalb dürfte man vor Allem prüfen, ob nicht unter den Harnpigmenten sich solche befinden, welche auf Epinephrin zurückzuführen sind.

In der That zeigt der als Uroerythrin bezeichnete, dem ziegelrothen Harnsedimente anhaftende Farbstoff einige der Reactionen des Epinephrins. Thudichum ${ }^{1}$ ) hat beobachtet, dass festes Uroerythrin durch Kalilauge sofort dụnkelgrün gefärbt wird, und Riva, Zoja und Garrod haben die Thatsache befestigt, dass auf Zusatz von viel Lauge zu einem an Uroerythrin reichen Harn ein schmutzig grüner Phosphatniederschlag entsteht. Garrod ${ }^{2}$ ) erwähnt auch, dass die amylalkoholische Lösung des Uroerythrins auf Zusatz von wenig Natronlauge eine rein grüne Färbung annimmt. Nun ist das Epinephrin das einzige bis jetzt bekannte Produkt des Thierkörpers, welches sich in seinem Verhalten in dieser Beziehung dem Uroerythrin an die Seite stellen lässt, denn wie ich schon mehrmals erwähnt habe, nimmt eine lösung des inactiven Epinephrins auf Zusatz von wenig Lauge eine rein grüne Färbung an. Riva ${ }^{3}$ ) beobachtete auch, dass manchmil eine Uroerythrinlösung auf Zusatz von Lauge violett, dann indigoblau, grün und darauf schnell farblos wird. Einen ähnlichen Farbenwandel kann man. auch bei der wirksamen Form des Epinephrins beobachten.

Versuche mit einer sehr geringen Menge eines von einem Schüler dargestellten Präparates von Uroerythrin, für dessen Rein-

1) Journ. chem. Soc. [2] 13. p. 399.

2) Journ. of Physiology.

3) Neubauer u. Vogel, Analyse des Harns. 10. Aufl. S. 585. 
heit ich nicht einstehen will, zeigten, dass es die Mandelin'sche Reaction sowie die mit concentrirter $\mathrm{H}_{2} \mathrm{SO}_{4}+\mathrm{KMnO}_{4}$ liefert und zwar so, dass sie von denen des Epinephrins nicht zu unterscheiden sind. Auch geben die rothen Harnsäuresedimente letztere Reaction in schönster Weise und liefern auch beim Schmelzen mit Kali ansehnliche Mengen von Skatol. Hierbei ist zu betonen, dass dem unter diesen Umständen erscheinenden Skatol keine Beweiskraft für seine Entstehung aus Uroerythrin ertheilt werden kann, da Zoja ${ }^{1}$ ) aus Urohämatoporphyrin auch Skatol erhalten hat.

Aus diesen Vorversuchen soll nur gefolgert werden, dass das Uroerythrin möglicher Weise ein Umwandlungsprodukt des im Thierkörper normal gebildeten Epinephrins ist. Die genannten Versuche ermuthigen zu einer weiteren Prüfung der Frage und es werden jetzt in meinem Laboratorium Versuche angestellt, grössere Mengen von Uroerythrin herzustellen, um weitere und genauere Vergleiche anstellen zu können.

Man wird leicht erkennen, dass die Kenntniss des Verhaltens des Epinephrins im normalen Gesundheitszustand $\mathrm{zu}$ interessanten Aufschlüssen in Betreff des Urins bei der Addisonschen Krankheit führen kann, dass also die Diagnose bei dieser Krankheit durch Harnuntersuchung möglicher Weise erleichtert werden könnte.

\section{Zusammenfassung.}

1. Der blutdrucksteigernde Bestandtheil der Nebenniere ist eine besondere unbeständige basische Substanz, deren procentische Zusammensetzung durch die Formel $\mathrm{C}_{17} \mathrm{H}_{15} \mathrm{NO}_{4}$ ausgedrückt wird und welche ich Epinephrin nenne.

2. Diese Substanz wurde aus den wässerigen Extracten jener Drüse als Benzoylverbindung isolirt und aus dieser wurden verschiedene physiologisch wirksame Salze genommen.

3. Die freie Base kann nicht ohne bedeutende Veränderung (Umlagerung zu einer wirkungslosen Substanz) und Verlust der physiologisch wirksamen Eigenschaften dargestellt werden.

1) Maly's Jahresb. f. Thierchemie. Bd. 23. s. 590 . 
4. Das Verhalten des Körpers bei der trockenen Destillation und in der Kalischmelze, die elementare Zusammensetzung, sowie das Verhalten $\mathrm{zu}$ verschiedenen Reagentien deuten auf die Alkaloidnatur desselben. In Bezug auf den Kerntypus darf man den Körper zu den Pyrrol-, resp. Skatolbasen zählen. Die 'Totalanzahl von (OH)-Gruppen steht noch nicht ganz fest, ebenfalls ist die Unterscheidung zwischen einer Aldehyd- oder Ketongruppe in seinem Molekül noch nicht vollendet und die Bindungsweise des Stickstoffs noch nicht sicher gestellt. Bei der Kalischmelze entstehen ansehnliche Mengen von Skatol.

5. Ein dunkles Pigment, Epinephrinsäure, entsteht stets, wenn Epinephrin mit verdünnten Alkalien behandelt wird. Ein zweites mit verdünnten, sowie mit stärkeren Alkalien erzeugtes Produkt ist basischer Natur von nicht scharf zu präcisirendem, aber coniin- oder pyridinähnlichem Geruch.

6. Die wirksamen Salze des Epinephrins haben eine markante Contractionswirkung auf die Blutgefässe bei localer Anwendung, haben einen schwach bitteren Geschmack und bringen in leichtem Grade Gefühlslosigkeit auf der Zunge hervor. Bei Einführung in den Kreislauf bringen diese Salze eine bedeutende und bei richtiger Anwendung eine lang andauernde Blutdrucksteigerung hervor. Trocken aufbewahrt, büssen alle Salze mit der Zeit sehr an ihrer Löslichkeit ein, was bis jetzt der Anwendung genannter Salze noch sehr im Wege steht. Sie erregen zuerst, dann lähmen sie die Athmung durch Wirkung auf die Centren. Erst später nach weiteren Gaben wird das Herz gelähmt. Die toxische und letale Dose liegt weit über derjenigen, bei der eine wesentliche physiologische Wirkung ohne Schaden erfolgt.

7. Im normalen Zustand des Thieres und des Menschen geht das Epinephrin möglicher Weise in den Harn als Uroerythrin über, welches die Eigenschaft hat, Harnsäuresedimenten eine Rosafärbung zu ertheilen.

Zum Schlusse ist es mir eine angenehme Pflicht, der Firma P. D. Armour u. Co. in Chicago meinen besten Dank auszusprechen für die grosse Liberalität, mit welcher diese Herren mir entgegengekommen sind, indem sie mir in den 
letzten drei Jahren wässerige Extracte, nach meiner Vorschrift aus mehreren Hundert Kilo frischer Nebennieren des Rindes dargestellt, freigebig geliefert haben. Auch gebührt mein Dank den Herren Dr. W. Jones und A. C. Crawford, dem ersteren für seine öfters geleistete Hülfe bei den chemischen Analysen, letzterem für seine stetige Unterstützung bei den physiologischen Versuchen dieser sowohl als meiner früheren Abhandlungen über diesen Gegenstand.

Baltimore, den 14. Juli 1899. 